\title{
AN INTERTWINING OPERATOR FOR THE GROUP $B_{2}$
}

\author{
CHARLES F. DUNKL \\ Department of Mathematics, PO Box 400137, University of Virginia, Charlottesville, VA 22904-4137 \\ e-mail: cfd5z@virginia.edu \\ URL: http://www.people.virginia.edu/ $c f d 5 z /$
}

(Received 4 August, 2006; revised 19 March, 2007; accepted 31 March, 2007)

\begin{abstract}
There is a commutative algebra of differential-difference operators, acting on polynomials on $\mathbb{R}^{2}$, associated with the reflection group $B_{2}$. This paper presents an integral transform which intertwines this algebra, allowing one free parameter, with the algebra of partial derivatives. The method of proof depends on properties of a certain class of balanced terminating hypergeometric series of ${ }_{4} F_{3}$-type. These properties are in the form of recurrence and contiguity relations and are proved herein.
\end{abstract}

2000 Mathematics Subject Classification. Primary 33C80, 33C20; Secondary 33C70, 43A80.

\section{Introduction.}

1.1. Overview. We construct an integral for the intertwining operator $V$ associated to the reflection group of type $B_{2}$ (order 8 ) acting on $\mathbb{R}^{2}$, with one parameter $\kappa$. For polynomials or adequately smooth functions in $x=\left(x_{1}, x_{2}\right)$ define the differential-difference operators:

$$
\begin{aligned}
T_{1} f(x)= & \frac{\partial}{\partial x_{1}} f(x)+\kappa_{1} \frac{f(x)-f\left(-x_{1}, x_{2}\right)}{x_{1}} \\
& +\kappa_{2} \frac{f(x)-f\left(x_{2}, x_{1}\right)}{x_{1}-x_{2}}+\kappa_{2} \frac{f(x)-f\left(-x_{2},-x_{1}\right)}{x_{1}+x_{2}}, \\
T_{2} f(x)= & \frac{\partial}{\partial x_{2}} f(x)+\kappa_{1} \frac{f(x)-f\left(x_{1},-x_{2}\right)}{x_{2}} \\
& +\kappa_{2} \frac{f(x)-f\left(x_{2}, x_{1}\right)}{x_{2}-x_{1}}+\kappa_{2} \frac{f(x)-f\left(-x_{2},-x_{1}\right)}{x_{1}+x_{2}} .
\end{aligned}
$$

These operators are special cases of those defined by the author in [2]. Their key property is commutativity, $T_{1} T_{2}=T_{2} T_{1}$. We deal only with the restricted case $\kappa_{1}=\kappa_{2}=\kappa$. The intertwining operator $V$ preserves the degree of homogeneous polynomials and satisfies $V\left(\frac{\partial}{\partial x_{i}} f\right)(x)=T_{i} V f(x)$ for $i=1$, 2, and $V 1=1$. The definition and existence of $V$ for $\kappa>0$ was shown in [3].

The easy example for this operator is furnished by the $\mathbb{Z}_{2}$ action $(x \longrightarrow-x)$ on $\mathbb{R}$. Let $\kappa$ be a parameter and define $T f(x)=f^{\prime}(x)+\kappa \frac{f(x)-f(-x)}{x}$ for a smooth function $f$ 
on $\mathbb{R}$. For $\kappa>0$ let

$$
V f(x):=B\left(\kappa, \frac{1}{2}\right)^{-1} \int_{-1}^{1} f(t x)(1+t)\left(1-t^{2}\right)^{\kappa-1} d t
$$

then $T V f(x)=V\left(\frac{d}{d x} f\right)(x)$ for any smooth function $f$. The attempt to find such formulae for reflection groups of higher rank starts by interpreting $x \longmapsto t x$ as a linear transformation (element of $M_{1}(\mathbb{R})$ ) on $\mathbb{R}$, and the measure

$$
d \mu(t):=B\left(\kappa, \frac{1}{2}\right)^{-1}\left(1-t^{2}\right)^{\kappa-1} d t,-1 \leq t \leq 1,
$$

as a $\mathbb{Z}_{2}$-invariant measure on a certain subset of $M_{1}(\mathbb{R})$; the term $1+t$ is explained by (very simple!) representation theory: if $f$ is even $(f(-x)=f(x))$ then $V f(x)=$ $\int_{-1}^{1} f(t x) d \mu(t)$ and if $f$ is odd $(f(-x)=-f(x))$ then $V f(x)=\int_{-1}^{1} f(t x) t d \mu(t)$. This program was carried out by the author for the group $S_{3}$ (symmetric group on 3 objects) in [4]. This paper establishes a criterion, valid for any finite reflection group, for an operator to be equal to $V$. The criterion is in terms of $\exp \left(\sum_{i=1}^{N} x_{i} y_{i}\right)$ for a reflection group acting on $\mathbb{R}^{N}$. When applied to the above formula for $V$ on $\mathbb{R}^{1}$ the criterion reduces to

$$
\int_{-1}^{1}(x y-x t y) e^{x t y}(1+t) d \mu(t)=\kappa \int_{-1}^{1} e^{x t y}((1+t)-(1-t)) d \mu(t),
$$

for arbitrary $x, y \in \mathbb{R}$. This identity obviously holds (integration-by-parts). Further details on postulating a formula for $V$ appropriate to the group $B_{2}$ in terms of a measure on $M_{2}(\mathbb{R})$ are to be found in Section 1.3. In [5] it was shown that $V$ exists and is one-to-one as a map on polynomials for any $\kappa$ except for the set $\left\{-\frac{m}{4}: m \in \mathbb{N}, \frac{m}{4} \notin \mathbb{Z}\right\}$ of singular values (for the case of $B_{2}$ ). Later Rösler [8] (see also [9]) proved that the functional $f \mapsto V f(x)$ is given by integration with respect to a positive measure, for each $x \in \mathbb{R}^{2} . \mathrm{Xu}[\mathbf{1 0}]$ found an intertwining transform for $B_{2}$ under restrictive conditions on degree and $\kappa_{1}, \kappa_{2}$.

There is a kernel which contains all the information about the action of $V$ on polynomials. For $x, y \in \mathbb{R}^{2}$ let $\langle x, y\rangle:=\sum_{i=1}^{2} x_{i} y_{i}$, then

$$
K(x, y):=V^{x}(\exp \langle x, y\rangle)
$$

is entire in $x$ and $y\left(V^{x}\right.$ acts on the variable $\left.x\right)$. Further let $K_{n}(x, y):=\frac{1}{n !} V^{x}\left(\langle x, y\rangle^{n}\right)$ for $n \in \mathbb{N}_{0}:=\{0,1,2, \ldots\}$. The symmetrized kernel $K^{0}$ is defined by

$$
K^{0}(x, y):=\frac{1}{8} \sum_{w \in B_{2}} K(x w, y)
$$

where the sum is over the eight elements of $B_{2}$. This kernel is also called a Bessel function because in the $\mathbb{Z}_{2}$ situation described above one has

$$
\begin{aligned}
\frac{1}{2} V^{x}\left(e^{x y}+e^{-x y}\right) & =\int_{-1}^{1} e^{x y t} d \mu(t)=\sum_{n=0}^{\infty} \frac{1}{\left(\kappa+\frac{1}{2}\right)_{n} n !}\left(\frac{x y}{2}\right)^{2 n} \\
& =\Gamma\left(\kappa+\frac{1}{2}\right)\left(\frac{x y}{2}\right)^{-\kappa+\frac{1}{2}} I_{\kappa-\frac{1}{2}}(x y), \text { for } x y>0
\end{aligned}
$$


one of the modified Bessel functions. The book of Dunkl and $\mathrm{Xu}$ [6] is a reference for differential-difference operators, the intertwining operator and the kernel $K(x, y)$ for any finite reflection group. Asymptotic formulae for the kernel are discussed in [9].

The author thanks the referee for useful remarks which helped improve the introduction and other details in the exposition.

1.2. The symplectic group integral. The starting point for the $S_{3}$ result was an integral formula of Harish-Chandra involving integration over a compact Lie group. This gives a special case ( $\kappa=1$ and symmetrized) of the intertwining operator for the associated Weyl group. We use an approach similar to that in [4, Section 2]. Consider $B_{2}$ as the Weyl group of the compact symplectic group $S p(2)$. This group can be described as the group of $2 \times 2$ unitary block matrices

$$
U=\left(\begin{array}{cc}
A & B \\
-\bar{B} & \bar{A}
\end{array}\right), U U^{*}=I
$$

where $A, B$ are $2 \times 2$ complex matrices. The subgroup

$$
T:=\left\{\operatorname{diag}\left(e^{\mathrm{i} \theta_{1}}, e^{\mathrm{i} \theta_{2}}, e^{-\mathrm{i} \theta_{1}}, e^{-\mathrm{i} \theta_{2}}\right): \theta_{1}, \theta_{2} \in \mathbb{R}\right\}
$$

is a maximal torus ("diag" denotes the $4 \times 4$ diagonal matrix with the specified entries). Identify $\mathbb{R}^{2}$ with the complexification of the Lie algebra of $T$ by the map $\delta: x \mapsto$ $2^{-1 / 2} \operatorname{diag}\left(x_{1}, x_{2},-x_{1},-x_{2}\right)$ (the purpose of the factor $2^{-1 / 2}$ is to get $\operatorname{Tr}(\delta(x) \delta(y))=$ $\langle x, y\rangle)$. The formula of Harish-Chandra (Helgason [7, p. 328]) specializes to

$$
\int_{S p(2)} \exp \left(\operatorname{Tr}\left(U \delta(x) U^{*} \delta(y)\right)\right) d m(U)=\frac{3}{2} \frac{\sum_{w \in B_{2}} \operatorname{det}(w) \exp (\langle x w, y\rangle)}{p(x) p(y)},
$$

where $d m(U)$ is normalized Haar measure on $S p(2)$ and $p(x)=x_{1} x_{2}\left(x_{1}^{2}-x_{2}^{2}\right)$. The right side of the formula is an expression for the kernel $K^{0}(x, y)$ at $\kappa=1$. Thus the left side suggests a construction of an integral formula for the intertwining operator. We compute

$$
\operatorname{Tr}\left(U \delta(x) U^{*} \delta(y)\right)=\sum_{i=1}^{2} \sum_{j=1}^{2} x_{i} y_{j}\left(\left|A_{j i}\right|^{2}-\left|B_{j i}\right|^{2}\right) .
$$

Thus one needs to integrate functions of the four variables $\left(\left|A_{j i}\right|^{2}-\left|B_{j i}\right|^{2}\right)$ with respect to Haar measure. One applies integration over the subgroup $S p(1)$ and the homogeneous space $S p(2) / S p(1)$. Since this only gives the $\kappa=1$ situation more experimentation is needed to make a conjecture about arbitrary $\kappa$. The measure that arises can be described in terms of trigonometric coordinates and a weight function. By direct polynomial calculation (using computer-assisted symbolic computation) we find the symmetrized kernel $K_{n}^{0}(x, y):=\frac{1}{8} \sum_{w \in B_{2}} V^{x}\langle x w, y\rangle^{n} / n$ ! for small $n(\leq 8)$ and try powers of the weight function to produce these values of $K_{n}^{0}(x, y)$. This approach, however, has as yet not produced a solution for the two-parameter situation. 
1.3. Group actions and the measure. The group $B_{2}$ is generated by the reflections $\sigma_{1}:=\left(\begin{array}{cc}-1 & 0 \\ 0 & 1\end{array}\right)$ and $\sigma_{2}:=\left(\begin{array}{ll}0 & 1 \\ 1 & 0\end{array}\right)$. The intertwining operator has the form

$$
\begin{aligned}
V f(x) & =\int_{\Omega} f(x \tau(q)) g(q) d \mu(q), \\
\tau(q) & :=\left(\begin{array}{ll}
q_{1} & q_{3} \\
q_{2} & q_{4}
\end{array}\right),
\end{aligned}
$$

where $\mu(q)$ is two-sided invariant for $B_{2}$ and $g(q)$ is invariant under the action $w: \tau(q) \rightarrow w \tau(q) w^{-1}$ for all $w \in B_{2}$. (Actually $g(q)$ involves one more variable of integration.) There are left and right representations of $B_{2}$ on $q$ (that is, $\mathbb{R}^{4}$ ), defined implicitly for $w \in B_{2}$ by

$$
\begin{aligned}
& \tau(q \lambda(w))=w^{-1} \tau(q), \\
& \tau(q \rho(w))=\tau(q) w
\end{aligned}
$$

For example $q \lambda\left(\sigma_{1}\right)=\left(-q_{1}, q_{2},-q_{3}, q_{4}\right)$ and $q \rho\left(\sigma_{2}\right)=\left(q_{3}, q_{4}, q_{1}, q_{2}\right)$. The invariance conditions are $d \mu(q \lambda(w))=d \mu(q \rho(w))=d \mu(q)$ and $g(q \lambda(w) \rho(w))=g(q)$ for all $w \in B_{2}$. It suffices to check invariance for the generating reflections; that is, $g$ and $\mu$ must be invariant under $q \mapsto q \lambda\left(\sigma_{1}\right) \rho\left(\sigma_{1}\right)=\left(q_{1},-q_{2},-q_{3}, q_{4}\right)$ and $q \mapsto$ $q \lambda\left(\sigma_{2}\right) \rho\left(\sigma_{2}\right)=\left(q_{4}, q_{3}, q_{2}, q_{1}\right)$; and, additionally, $\mu$ must be invariant under $q \mapsto$ $q \lambda\left(\sigma_{1}\right)=\left(-q_{1}, q_{2},-q_{3}, q_{4}\right)$ and $q \mapsto q \lambda\left(\sigma_{2}\right)=\left(q_{2}, q_{1}, q_{4}, q_{3}\right)$.

The measure $\mu$ is actually an integral over six variables: let

$$
\begin{aligned}
& q_{1}=u \cos \psi_{1}, \\
& q_{2}=(1-u) \cos \psi_{2}, \\
& q_{3}=(1-u)\left(\cos \psi_{2} \cos \theta+\sin \psi_{2} \sin \theta \cos \phi_{2}\right), \\
& q_{4}=u\left(\cos \psi_{1} \cos \theta+\sin \psi_{1} \sin \theta \cos \phi_{1}\right)
\end{aligned}
$$

the region $\Omega$ of integration is $0 \leq u \leq 1,0 \leq \theta, \phi_{i}, \psi_{i} \leq \pi(i=1,2)$. For $\kappa>\frac{1}{2}$ the measure is

$$
d \mu(q)=c_{\kappa}\left(u(1-u) \sin \psi_{1} \sin \psi_{2} \sin \theta\right)^{2 \kappa-1}\left(\sin \phi_{1} \sin \phi_{2}\right)^{2 \kappa-2} d u d \psi_{1} d \psi_{2} d \theta d \phi_{1} d \phi_{2},
$$

where the condition $\int_{\Omega} d \mu=1$ determines the normalizing constant $c_{\kappa}$. We can now state the main result for homogeneous polynomials, by use of the differential operator

$$
D_{0}=\left(q_{1}+q_{4}\right)\left(\frac{\partial}{\partial q_{1}}+\frac{\partial}{\partial q_{4}}\right)-\left(q_{2}-q_{3}\right)\left(\frac{\partial}{\partial q_{2}}-\frac{\partial}{\partial q_{3}}\right) .
$$

THEOREM 1. Suppose that $f(x)$ is a homogeneous polynomial in $x$ of degree $n$. If $n$ is odd, then

$$
V f(x)=\int_{\Omega} 2\left(q_{1}+q_{4}\right) f(x \tau(q)) d \mu(q) .
$$

If $n$ is even, then

$$
V f(x)=\int_{\Omega}\left\{1+q_{1} q_{4}-q_{2} q_{3}+\frac{1}{4 \kappa+n} D_{0}\right\} f(\tau(q) x) d \mu(q) .
$$


Further, the Bessel function $K^{0}(x, y)=\frac{1}{8} \sum_{w \in B_{2}} K(x w, y)$ is given by the positive integral

$$
K^{0}(x, y)=\int_{\Omega} \exp (\langle x \tau(q), y\rangle) d \mu(q)
$$

We shall consider the relation to the representations of $B_{2}$ and the techniques of proving the formulae in the following sections. The required invariance properties of $\mu$ are made clear by a change-of-variables.

LEMMA 1. In terms of $q, \theta, u$ and the auxiliary variables

$$
\begin{aligned}
z_{1}\left(q_{1}, q_{4}, u, \theta\right) & :=\frac{u^{2} \sin ^{2} \theta-q_{1}^{2}-q_{4}^{2}+2 q_{1} q_{4} \cos \theta}{\sin ^{2} \theta} \\
z_{2}\left(q_{2}, q_{3}, u, \theta\right) & :=\frac{(1-u)^{2} \sin ^{2} \theta-q_{2}^{2}-q_{3}^{2}+2 q_{2} q_{3} \cos \theta}{\sin ^{2} \theta}
\end{aligned}
$$

the measure is given by

$$
d \mu=c_{\kappa}\left(z_{1} z_{2}\right)^{\kappa-3 / 2}(\sin \theta)^{2 \kappa-3} d q_{1} d q_{2} d q_{3} d q_{4} d u d \theta,
$$

and the region of integration $\Omega_{1}$ is implicitly defined by $z_{1} \geq 0, z_{2} \geq 0,0 \leq \theta \leq \pi, 0 \leq$ $u \leq 1$.

Proof. In terms of $q_{1}, q_{2}, q_{3}, q_{4}, u, \theta$ the Jacobian is

$$
J=\frac{\partial\left(q_{1}, q_{2}, q_{3}, q_{4}, u, \theta\right)}{\partial\left(\phi_{1}, \phi_{2}, \psi_{1}, \psi_{2}, u, \theta\right)}=\left(u(1-u) \sin \psi_{1} \sin \psi_{2} \sin \theta\right)^{2} \sin \phi_{1} \sin \phi_{2} .
$$

Then

$$
d \mu=c_{\kappa}\left(u(1-u) \sin \psi_{1} \sin \psi_{2} \sin \phi_{1} \sin \phi_{2} \sin \theta\right)^{2 \kappa-3} J d u d \psi_{1} d \psi_{2} d \theta d \phi_{1} d \phi_{2} .
$$

Observe that $u \sin \psi_{1} \sin \theta \cos \phi_{1}=q_{4}-q_{1} \cos \theta, u^{2} \sin ^{2} \psi_{1}=u^{2}-q_{1}^{2}$, and so

$$
\begin{aligned}
\sin ^{2} \phi_{1} & =1-\left(\frac{q_{4}-q_{1} \cos \theta}{u \sin \psi_{1} \sin \theta}\right)^{2}, \\
\left(u \sin \psi_{1} \sin \phi_{1}\right)^{2} & =\left(u^{2}-q_{1}^{2}\right)-\frac{\left(q_{4}-q_{1} \cos \theta\right)^{2}}{\sin ^{2} \theta} \\
& =z_{1}\left(q_{1}, q_{4}, u, \theta\right) .
\end{aligned}
$$

Similarly

$$
\begin{aligned}
\left((1-u) \sin \psi_{2} \sin \phi_{2}\right)^{2} & =\left((1-u)^{2}-q_{2}^{2}\right)-\frac{\left(q_{3}-q_{2} \cos \theta\right)^{2}}{\sin ^{2} \theta} \\
& =z_{2}\left(q_{2}, q_{3}, u, \theta\right)
\end{aligned}
$$

Thus $d \mu=c_{\kappa}\left(z_{1} z_{2}\right)^{\kappa-3 / 2}(\sin \theta)^{2 \kappa-3} d q_{1} d q_{2} d q_{3} d q_{4} d u d \theta$.

The measure $\mu$ is invariant under the transpositions $\left(q_{1}, q_{4}\right),\left(q_{2}, q_{3}\right)$, and the involutions $q \mapsto q \lambda\left(\sigma_{1}\right)=\left(q_{2}, q_{1}, q_{4}, q_{3}\right)$ and $q \mapsto q \lambda\left(\sigma_{1}\right)=\left(-q_{1},-q_{2}, q_{3}, q_{4}\right)$ because 
of the equations

$$
\begin{aligned}
z_{1}\left(q_{2}, q_{3}, u, \theta\right) z_{2}\left(q_{1}, q_{4}, u, \theta\right) & =z_{1}\left(q_{1}, q_{4}, 1-u, \theta\right) z_{2}\left(q_{2}, q_{3}, 1-u, \theta\right), \\
z_{1}\left(-q_{1}, q_{4}, u, \theta\right) z_{2}\left(-q_{2}, q_{3}, u, \theta\right) & =z_{1}\left(q_{1}, q_{4}, u, \pi-\theta\right) z_{2}\left(q_{2}, q_{3}, u, \pi-\theta\right) .
\end{aligned}
$$

Similarly there is invariance under the right action of $B_{2}$; that is, $q \mapsto q \rho\left(\sigma_{i}\right), i=1,2$.

The following will be used throughout.

Notation 1. Suppose that $\alpha \in \mathbb{N}_{0}^{4}$, Then the corresponding monomial is $q^{\alpha}:=$ $q_{1}^{\alpha_{1}} q_{2}^{\alpha_{2}} q_{3}^{\alpha_{3}} q_{4}^{\alpha_{4}}$ and $q^{\alpha}$ is of degree $|\alpha|:=\sum_{i=1}^{4} \alpha_{i}$. Also set

$$
\begin{aligned}
& b_{0}:=\left(\alpha_{2}+\alpha_{3}\right) / 2, \\
& b_{1}:=\left(\alpha_{1}+\alpha_{4}\right) / 2, \\
& b_{2}:=\left(\alpha_{2}+\alpha_{4}\right) / 2, \\
& b_{3}:=\left(\alpha_{3}+\alpha_{4}\right) / 2,
\end{aligned}
$$

and let $s\left(\alpha_{1}, \alpha_{2}, \alpha_{3}, \alpha_{4}\right):=\int_{\Omega} q^{\alpha} d \mu(q)$.

By a parity argument the nonzero integrals occur only for integer values of the $b_{i}$.

PROPOSITION 1. The normalizing constant is

$$
c_{\kappa}=4^{\kappa-1} \frac{(2 \kappa-1)^{2} \Gamma\left(2 \kappa+\frac{1}{2}\right)}{\pi^{5 / 2} \Gamma(\kappa)^{2}},
$$

and, for $\alpha \in \mathbb{N}_{0}^{4}$, if $\alpha_{1} \equiv \alpha_{2} \equiv \alpha_{3} \equiv \alpha_{4} \bmod 2$ (all even or all odd) then

$$
\begin{aligned}
s\left(\alpha_{1}, \alpha_{2}, \alpha_{3}, \alpha_{4}\right)= & \frac{(2 \kappa)_{2 b_{1}}(2 \kappa)_{2 b_{0}}\left(\frac{1}{2}\right)_{b_{1}}\left(\frac{1}{2}\right)_{b_{0}}\left(\frac{1}{2}\right)_{b_{3}}}{(4 \kappa)_{2 b_{1}+2 b_{0}}\left(\kappa+\frac{1}{2}\right)_{b_{1}}\left(\kappa+\frac{1}{2}\right)_{b_{0}}\left(\kappa+\frac{1}{2}\right)_{b_{3}}} \\
& \times \sum_{i=0}^{\left\lfloor\alpha_{4} / 2\right\rfloor} \sum_{j=0}^{\left\lfloor\alpha_{3} / 2\right\rfloor} \frac{\left(-\alpha_{4}\right)_{2 i}\left(-\alpha_{3}\right)_{2 j}(\kappa)_{i+j}}{i ! j !\left(\frac{1}{2}-b_{1}\right)_{i}\left(\frac{1}{2}-b_{0}\right)_{j}\left(\frac{1}{2}-b_{3}\right)_{i+j}} 2^{-2 i-2 j} .
\end{aligned}
$$

Otherwise $s\left(\alpha_{1}, \alpha_{2}, \alpha_{3}, \alpha_{4}\right)=0$.

Proof. Expand $q^{\alpha}$ in terms of $u, \theta, \psi_{1}, \psi_{2}, \phi_{1}, \phi_{2}$ with the binomial theorem and collect terms. The result is

$$
\begin{aligned}
s\left(\alpha_{1}, \alpha_{2}, \alpha_{3}, \alpha_{4}\right)= & c_{\kappa} \sum_{j=0}^{\alpha_{3}} \sum_{i=0}^{\alpha_{4}}\left(\begin{array}{c}
\alpha_{3} \\
j
\end{array}\right)\left(\begin{array}{c}
\alpha_{4} \\
i
\end{array}\right) \int_{0}^{1} u^{\alpha_{1}+\alpha_{4}+2 \kappa-1}(1-u)^{\alpha_{2}+\alpha_{3}+2 \kappa-1} d u \\
& \times \int_{0}^{\pi}\left(\cos \psi_{1}\right)^{\alpha_{1}+\alpha_{4}-i}\left(\sin \psi_{1}\right)^{i+2 \kappa-1} d \psi_{1} \int_{0}^{\pi}\left(\cos \phi_{1}\right)^{i}\left(\sin \phi_{1}\right)^{2 \kappa-2} d \phi_{1} \\
& \times \int_{0}^{\pi}\left(\cos \psi_{2}\right)^{\alpha_{2}+\alpha_{3}-j}\left(\sin \psi_{2}\right)^{j+2 \kappa-1} d \psi_{2} \int_{0}^{\pi}\left(\cos \phi_{2}\right)^{j}\left(\sin \phi_{2}\right)^{2 \kappa-2} d \phi_{2} \\
& \times \int_{0}^{\pi}(\cos \theta)^{\alpha_{3}+\alpha_{4}-i-j}(\sin \theta)^{i+j+2 \kappa-1} d \theta .
\end{aligned}
$$

Recall that $\int_{0}^{\pi} \cos ^{n} \theta \sin ^{\lambda} \theta d \theta$ equals zero if $n$ is odd and equals $B\left(\frac{n+1}{2}, \frac{\lambda+1}{2}\right)$ if $n$ is even, for $n \in \mathbb{N}_{0}$ and $\lambda>-1$. For the respective integrals to be nonzero $i$ and $j$ must be even because of the $\phi_{1}$ and $\phi_{2}$ integrals and hence $\alpha_{2}+\alpha_{3}, \alpha_{1}+\alpha_{4}, \alpha_{3}+\alpha_{4}$ must be even 
(in the $\psi_{1}, \psi_{2}, \theta$ integrals). We replace $i, j$ by $2 i, 2 j$ respectively and assume that the entries of $\alpha$ are all even or all odd. Then

$$
\begin{aligned}
s\left(\alpha_{1}, \alpha_{2}, \alpha_{3}, \alpha_{4}\right)= & c_{\kappa} \sum_{j=0}^{\left\lfloor\alpha_{3} / 2\right\rfloor} \sum_{i=0}^{\left\lfloor\alpha_{4} / 2\right\rfloor}\left(\begin{array}{l}
\alpha_{3} \\
2 j
\end{array}\right)\left(\begin{array}{c}
\alpha_{4} \\
2 i
\end{array}\right) B\left(\alpha_{1}+\alpha_{4}+2 \kappa, \alpha_{2}+\alpha_{3}+2 \kappa\right) \\
& \times B\left(\frac{\alpha_{1}+\alpha_{4}+1}{2}-i, i+\kappa\right) B\left(i+\frac{1}{2}, \kappa-\frac{1}{2}\right) \\
& \times B\left(\frac{\alpha_{2}+\alpha_{3}+1}{2}-j, j+\kappa\right) B\left(j+\frac{1}{2}, \kappa-\frac{1}{2}\right) \\
& \times B\left(\frac{\alpha_{3}+a_{4}+1}{2}-i-j, i+j+\kappa\right) .
\end{aligned}
$$

We set $\alpha=0$ to find the normalizing constant. Indeed

$$
c_{\kappa}^{-1}=B(2 \kappa, 2 \kappa) B\left(\frac{1}{2}, \kappa\right)^{3} B\left(\frac{1}{2}, \kappa-\frac{1}{2}\right)^{2} .
$$

The stated value follows from the duplication formula. The following ratios are typical in the calculation.

$$
\begin{aligned}
\frac{B\left(i+\frac{1}{2}, \kappa-\frac{1}{2}\right)}{B\left(\frac{1}{2}, \kappa-\frac{1}{2}\right)} & =\frac{\Gamma\left(i+\frac{1}{2}\right) \Gamma\left(\kappa-\frac{1}{2}\right) \Gamma(\kappa)}{\Gamma(\kappa+i) \Gamma\left(\frac{1}{2}\right) \Gamma\left(\kappa-\frac{1}{2}\right)}=\frac{\left(\frac{1}{2}\right)_{i}}{(\kappa)_{i}} \\
\frac{B\left(b_{0}+\frac{1}{2}-i, i+\kappa\right)}{B\left(\frac{1}{2}, \kappa\right)} & =\frac{\Gamma\left(b_{0}+\frac{1}{2}-i\right) \Gamma(i+\kappa) \Gamma\left(\kappa+\frac{1}{2}\right)}{\Gamma\left(b_{0}+\frac{1}{2}+\kappa\right) \Gamma\left(\frac{1}{2}\right) \Gamma(\kappa)}=\frac{(\kappa)_{i}\left(\frac{1}{2}\right)_{b_{0}-i}}{\left(\kappa+\frac{1}{2}\right)_{b_{0}}} .
\end{aligned}
$$

Thus

$$
\begin{aligned}
s\left(\alpha_{1}, \alpha_{2}, \alpha_{3}, \alpha_{4}\right)= & \sum_{i=0}^{\left\lfloor\alpha_{3} / 2\right\rfloor} \sum_{j=0}^{\left\lfloor\alpha_{4} / 2\right\rfloor}\left(\begin{array}{c}
\alpha_{3} \\
2 i
\end{array}\right)\left(\begin{array}{c}
\alpha_{4} \\
2 j
\end{array}\right) \frac{(2 \kappa)_{\alpha_{1}+\alpha_{4}}(2 \kappa)_{\alpha_{2}+\alpha_{3}}}{(4 \kappa)_{\alpha_{1}+\alpha_{2}+\alpha_{3}+\alpha_{4}}} \\
& \times \frac{\left(\frac{1}{2}\right)_{i}\left(\frac{1}{2}\right)_{b_{0}-i}\left(\frac{1}{2}\right)_{j}\left(\frac{1}{2}\right)_{b_{1}-j}\left(\frac{1}{2}\right)_{b_{3}-i-j}(\kappa)_{i+j}}{\left(\kappa+\frac{1}{2}\right)_{b_{0}}\left(\kappa+\frac{1}{2}\right)_{b_{1}}\left(\kappa+\frac{1}{2}\right)_{b_{3}}} .
\end{aligned}
$$

To finish the proof, write $\left(\begin{array}{c}\alpha_{4} \\ 2 i\end{array}\right)=\left(-\alpha_{4}\right)_{2 i} /\left(2^{2 i} i !\left(\frac{1}{2}\right)_{i}\right),\left(\frac{1}{2}\right)_{b_{0}-i}=(-1)^{i}\left(\frac{1}{2}\right)_{b_{0}} /\left(\frac{1}{2}-b_{0}\right)_{i}$ and similarly for the other terms.

It is clear that the symmetry $s\left(\alpha_{1}, \alpha_{2}, \alpha_{3}, \alpha_{4}\right)=s\left(\alpha_{2}, \alpha_{1}, \alpha_{4}, \alpha_{3}\right)$ holds (as well as $\left.s\left(\alpha_{1}, \alpha_{2}, \alpha_{3}, \alpha_{4}\right)=s\left(\alpha_{1}, \alpha_{3}, \alpha_{2}, \alpha_{4}\right)\right)$, as implied by Lemma 1 . Some other symmetries will be shown later. Singular values are numerical values of $\kappa$ for which the intertwining operator does not exist. By the general theory of singular values [5] specialized to $B_{2}$ they consist of $-\frac{1}{2}-\mathbb{N}_{0} \cup\left(-\frac{1}{4}-\mathbb{N}_{0} \cup-\frac{3}{4}-\mathbb{N}_{0}\right)$. The denominators $\left(\kappa+\frac{1}{2}\right)_{b_{i}}$ in $s\left(\alpha_{1}, \alpha_{2}, \alpha_{3}, \alpha_{4}\right)$ correspond to the first subset. For the second subset consider

$$
\begin{aligned}
\frac{(2 \kappa)_{2 b_{1}}(2 \kappa)_{2 b_{0}}}{(4 \kappa)_{2 b_{1}+2 b_{0}}} & =2^{-2 b_{1}-2 b_{0}} \frac{(2 \kappa)_{2 b_{1}}(2 \kappa)_{2 b_{0}}}{(2 \kappa)_{b_{1}+b_{0}}\left(2 \kappa+\frac{1}{2}\right)_{b_{1}+b_{0}}} \\
& =2^{-2 b_{1}-2 b_{0}} \frac{\left(2 \kappa+b_{1}+b_{0}\right)_{b_{1}-b_{0}}(2 \kappa)_{2 b_{0}}}{\left(2 \kappa+\frac{1}{2}\right)_{b_{1}+b_{0}}}
\end{aligned}
$$

if $b_{1} \geq b_{0}$. There is a similar expression if $b_{0} \geq b_{1}$. 
To see some of the complexity of this integral, consider the reduced form

$$
\begin{aligned}
& \int_{\Omega} f\left(q_{1}, q_{2}\right) d \mu(q) \\
& \quad=c \int_{0}^{1} \int_{0}^{\pi} \int_{0}^{\pi} f\left(u \cos \psi_{1},(1-u) \cos \psi_{2}\right)\left(u(1-u) \sin \psi_{1} \sin \psi_{2}\right)^{2 \kappa-1} d \psi_{1} d \psi_{2} d u
\end{aligned}
$$

with a constant $c$ depending on $\kappa$. This is not reducible to an ordinary double integral without the use of a nonelementary integral. Indeed,

$$
\begin{aligned}
\int_{\Omega} f\left(q_{1}, q_{2}\right) d \mu(q) & =\iint_{\left|q_{1}\right|+\left|q_{2}\right| \leq 1} f\left(q_{1}, q_{2}\right) E_{\kappa}\left(q_{1}, q_{2}\right) d q_{1} d q_{2}, \\
E_{\kappa}\left(q_{1}, q_{2}\right) & =c \int_{\left|q_{1}\right|}^{1-\left|q_{2}\right|}\left\{\left(u^{2}-q_{1}^{2}\right)\left((1-u)^{2}-q_{2}^{2}\right)\right\}^{\kappa-1} d u .
\end{aligned}
$$

1.4. Single sum formula and hypergeometric functions. Even though our evaluation of $s\left(\alpha_{1}, \alpha_{2}, \alpha_{3}, \alpha_{4}\right)$ required a six-variable integral the value can be expressed as a single sum in terms of a terminating balanced ${ }_{4} F_{3}$ hypergeometric series.

Write

$$
s\left(\alpha_{1}, \alpha_{2}, \alpha_{3}, \alpha_{4}\right)=\frac{(2 \kappa)_{2 b_{1}}(2 \kappa)_{2 b_{0}}}{(4 \kappa)_{2 b_{1}+2 b_{0}}} s^{\prime}\left(\alpha_{1}, \alpha_{2}, \alpha_{3}, \alpha_{4}\right)
$$

Then $s^{\prime}$ satisfies the recurrence

$$
\begin{aligned}
& \alpha_{1} \alpha_{4}\left(\kappa+\frac{1}{2}\left(\alpha_{2}+\alpha_{3}+1\right)\right) s^{\prime}\left(\alpha_{1}-1, \alpha_{2}+1, \alpha_{3}+1, \alpha_{4}-1\right) \\
& \quad+\frac{1}{2}\left(\alpha_{2} \alpha_{3}\left(\alpha_{1}+\alpha_{4}+1\right)-\alpha_{1} \alpha_{4}\left(\alpha_{2}+\alpha_{3}+1\right)\right) s^{\prime}\left(\alpha_{1}, \alpha_{2}, \alpha_{3}, \alpha_{4}\right) \\
& =\alpha_{2} \alpha_{3}\left(\kappa+\frac{1}{2}\left(\alpha_{1}+\alpha_{4}+1\right)\right) s^{\prime}\left(\alpha_{1}+1, \alpha_{2}-1, \alpha_{3}-1, \alpha_{4}+1\right) .
\end{aligned}
$$

There is a good reason why this formula appears here. The intertwining operator will be described as a linear functional on the space of polynomials in $q$, applied to polynomials in $x \tau(q)=\left(x_{1} q_{1}+x_{2} q_{2}, x_{1} q_{3}+x_{2} q_{4}\right)$. The coefficient of $x_{1}^{a+b-c} x_{2}^{c}$ in the expansion of $\left(x_{1} q_{1}+x_{2} q_{2}\right)^{a}\left(x_{1} q_{3}+x_{2} q_{4}\right)^{b}$ is as follows.

$$
P_{a, b}^{c}(q)=\sum_{i=\max (0, c-a)}^{\min (b, c)}\left(\begin{array}{c}
a \\
c-i
\end{array}\right)\left(\begin{array}{l}
b \\
i
\end{array}\right) q_{1}^{a-c+i} q_{2}^{c-i} q_{3}^{b-i} q_{4}^{i} .
$$

The need to integrate these polynomials motivated the examination of and experimentation with $s(a-c+i, c-i, b-i, i)$ as a function of $i$. This led to the discovery of the recurrence which in turn suggested that there might be a single-sum form of $s$. It turns out that the proof of the recurrence actually uses the single sum, a terminating hypergeometric series of ${ }_{4} F_{3}$-type (the argument looks circular, but the proof of the single sum does not use the recurrence). 
Proposition 2. Suppose that $\alpha_{4}=0$ and $\alpha_{i}=2 \beta_{i}$ for $1 \leq i \leq 3$, We have

$$
\begin{aligned}
s^{\prime}\left(2 \beta_{1}, 2 \beta_{2}, 2 \beta_{3}, 0\right) & =\frac{\left(\frac{1}{2}\right)_{\beta_{1}}\left(\frac{1}{2}\right)_{\beta_{2}}\left(\frac{1}{2}\right)_{\beta_{3}}}{\left(\kappa+\frac{1}{2}\right)_{\beta_{1}}\left(\kappa+\frac{1}{2}\right)_{\beta_{2}}\left(\kappa+\frac{1}{2}\right)_{\beta_{3}}} \\
& =s^{\prime}\left(0,2 \beta_{2}, 2 \beta_{3}, 2 \beta_{1}\right),
\end{aligned}
$$

and the value is symmetric in $\beta_{1}, \beta_{2}, \beta_{3}$.

Proof. Indeed the sum in $s^{\prime}\left(2 \beta_{1}, 2 \beta_{2}, 2 \beta_{3}, 0\right)$ equals

$$
\begin{aligned}
& \sum_{j=0}^{\beta_{3}} \frac{\left(-\beta_{3}\right)_{j}\left(\frac{1}{2}-\beta_{3}\right)_{j}(\kappa)_{j}}{j !\left(\frac{1}{2}-\beta_{2}-\beta_{3}\right)_{j}\left(\frac{1}{2}-\beta_{3}\right)_{j}} \\
& \quad=\frac{\left(\frac{1}{2}-\beta_{2}-\beta_{3}-\kappa\right)_{\beta_{3}}}{\left(\frac{1}{2}-\beta_{2}-\beta_{3}\right)_{\beta_{3}}}=\frac{\left(\kappa+\beta_{2}+\frac{1}{2}\right)_{\beta_{3}}}{\left(\frac{1}{2}+\beta_{2}\right)_{\beta_{3}}} \\
& \quad=\frac{\left(\kappa+\frac{1}{2}\right)_{\beta_{2}+\beta_{3}}\left(\frac{1}{2}\right)_{\beta_{2}}}{\left(\kappa+\frac{1}{2}\right)_{\beta_{2}}\left(\frac{1}{2}\right)_{\beta_{2}+\beta_{3}}}
\end{aligned}
$$

(by the Chu-Vandermonde sum) and this proves the first equation. The second equation follows from the symmetry of Lemma 1 . The sum in $s^{\prime}\left(0,2 \beta_{2}, 2 \beta_{3}, 2 \beta_{1}\right)$ can also be found directly, first summing over $0 \leq i \leq \beta_{1}$ and using similar arguments as in the first equation.

Recall the notations $b_{0}:=\left(\alpha_{2}+\alpha_{3}\right) / 2$ and $b_{i}:=\left(\alpha_{i}+\alpha_{4}\right) / 2$ for $i=1,2,3$.

THEOREM 2. Suppose that $\alpha \in \mathbb{N}_{0}^{4}$ and $\alpha_{1} \equiv \alpha_{2} \equiv \alpha_{3} \equiv \alpha_{4} \bmod 2$. Then

$$
\begin{aligned}
s^{\prime}\left(\alpha_{1}, \alpha_{2}, \alpha_{3}, \alpha_{4}\right)= & \frac{\left(\frac{1}{2}\right)_{b_{1}}\left(\frac{1}{2}\right)_{b_{2}}\left(\frac{1}{2}\right)_{b_{3}}}{\left(\kappa+\frac{1}{2}\right)_{b_{1}}\left(\kappa+\frac{1}{2}\right)_{b_{2}}\left(\kappa+\frac{1}{2}\right)_{b_{3}}} \\
& \times \sum_{i=0}^{\left\lfloor\alpha_{4} / 2\right\rfloor} \frac{\left(-\frac{\alpha_{4}}{2}\right)_{i}\left(\frac{1-\alpha_{4}}{2}\right)_{i}(\kappa)_{i}\left(-\kappa-b_{1}-b_{0}\right)_{i}}{i !\left(\frac{1}{2}-b_{1}\right)_{i}\left(\frac{1}{2}-b_{2}\right)_{i}\left(\frac{1}{2}-b_{3}\right)_{i}} .
\end{aligned}
$$

This is a balanced terminating ${ }_{4} F_{3}$ series.

The proof of this key result is in Section 3. To say that a hypergeometric series is balanced means that the sum of the denominator parameters equals one plus the sum of the numerator parameters; the property is also called Saalschützian. The particular choice of parameters for this ${ }_{4} F_{3}$ series will appear often in the sequel and so we make the following definition.

Definition 1. For $n \in \mathbb{N}_{0}$ and free parameters $u, v_{1}, v_{2}, v_{3}$ let

$$
F\left(n ; u, v_{1}, v_{2}, v_{3}\right)={ }_{4} F_{3}\left(\begin{array}{c}
-\frac{n}{2}, \frac{1-n}{2}, u,-u-v_{1}-v_{2}-v_{3} \\
\frac{1}{2}-v_{1}-n, \frac{1}{2}-v_{2}, \frac{1}{2}-v_{3} ; 1
\end{array}\right) .
$$

This is a balanced terminating hypergeometric function. 
This is not the generic balanced ${ }_{4} F_{3}$-series (which has 6 free parameters) because of the parameters $\left(-\frac{n}{2}, \frac{1-n}{2}\right)$. With this notation the Theorem can be restated as follows.

THEOREM 3. For $\alpha_{1} \equiv \alpha_{2} \equiv \alpha_{3} \equiv \alpha_{4} \bmod 2$ the following single-sum expression is valid:

$$
\begin{aligned}
s(\alpha)= & \frac{(2 \kappa)_{\alpha_{1}+\alpha_{4}}(2 \kappa)_{\alpha_{2}+\alpha_{3}}}{(4 \kappa)_{|\alpha|}} \frac{\left(\frac{1}{2}\right)_{b_{1}}\left(\frac{1}{2}\right)_{b_{2}}\left(\frac{1}{2}\right)_{b_{3}}}{\left(\kappa+\frac{1}{2}\right)_{b_{1}}\left(\kappa+\frac{1}{2}\right)_{b_{2}}\left(\kappa+\frac{1}{2}\right)_{b_{3}}} \\
& \times F\left(\alpha_{4} ; \kappa, \frac{1}{2}\left(\alpha_{1}-\alpha_{4}\right), \frac{1}{2}\left(\alpha_{2}+\alpha_{4}\right), \frac{1}{2}\left(\alpha_{3}+\alpha_{4}\right)\right) .
\end{aligned}
$$

1.5. Contents. In Section 2 we describe the representation-theoretic implications of the invariance conditions, present an overview of the method of proving that a given linear functional on polynomials in $q$ produces the intertwining operator and then give the actual proof. Some ingredients of the proofs depend on contiguity relations for the function $F$. Also there is a purely integral form of $V$ not involving the degree of polynomials.

Section 3 contains the proof of the single-sum result. That relies on classical transformations of hypergeometric series, and the (computer-assisted) proofs of the required contiguity relations of $F$. There are closing comments in Section 4 .

\section{The intertwining operator.}

2.1. Invariants and representations of $B_{2}$. As we suggested in the discussion of the $\mathbb{Z}_{2}$-intertwining operator, the integral for $V$ should consist of an invariant measure multiplied by a sum of terms, one for each irreducible representation of the group. The group $B_{2}$ acts on polynomials, $\mathbb{R}\left[x_{1}, x_{2}\right]$, by $R(w) p(x):=p(x w)$ (for $x \in \mathbb{R}^{2}, w \in B_{2}$ ). The irreducible representations of $B_{2}$ occur in this space as follows: there is one of degree 2 , which is realized in each space of polynomials homogeneous of degree $2 n-1$ as $n$ isomorphic copies

$$
\left\{c_{0} x_{1}^{2 n-1-j} x_{2}^{j}+c_{1} x_{1}^{j} x_{2}^{2 n-1-j}: 0 \leq j \leq n-1\right\}
$$

for $n=1,2, \ldots$; there are four nonequivalent one-dimensional representations:

(1) invariants: $x_{1}^{2 n-2 j} x_{2}^{2 j}+x_{1}^{2 j} x_{2}^{2 n-2 j}, 0 \leq j \leq n$;

(2) determinant: $x_{1}^{2 n-1-2 j} x_{2}^{2 j+1}-x_{1}^{2 j+1} x_{2}^{2 n-1-2 j}, 0 \leq j \leq\left\lfloor\frac{n}{2}\right\rfloor-1$ and $2 n \geq 4$;

(3) type 1: $x_{1}^{2 n-1-2 j} x_{2}^{2 j+1}+x_{1}^{2 j+1} x_{2}^{2 n-1-2 j}, 0 \leq j \leq\left\lfloor\frac{n-1}{2}\right\rfloor$ and $2 n \geq 2$;

(4) type 2: $x_{1}^{2 n-2 j} x_{2}^{2 j}-x_{1}^{2 j} x_{2}^{2 n-2 j}, 0 \leq j \leq\left\lfloor\frac{n-1}{2}\right\rfloor$ and $2 n \geq 2$.

If $p(x)$ is a polynomial of type 1 , then $p\left(x \sigma_{1}\right)=-p(x), p\left(x \sigma_{2}\right)=p(x)$; if $p(x)$ is of type 2 , then $p\left(x \sigma_{1}\right)=p(x), p\left(x \sigma_{2}\right)=-p(x)$. This is the reason for naming types. If $p(x)$ is of determinant type then $p\left(x \sigma_{1}\right)=-p(x), p\left(x \sigma_{2}\right)=-p(x)$. Consider polynomials in $q$ invariant under $q \mapsto q \lambda(w) \rho(w), w \in B_{2}$; this kind of invariance is required in the formula for $V$ because of the commutation $V(R(w) f)(x)=V f(x w), w \in B_{2}$. It is easy to compute the Poincaré series for the ring of invariants (graded by degree); namely 
$\frac{1+z^{3}}{(1-z)\left(1-z^{2}\right)^{3}}$. We then describe the ring as

$$
\left(1 \oplus\left(q_{1}-q_{4}\right)\left(q_{2}^{2}-q_{3}^{2}\right)\right) \mathbb{R}\left[q_{1}+q_{4}, q_{1}^{2}+q_{4}^{2}, q_{2}^{2}+q_{3}^{2}, q_{2} q_{3}\right] .
$$

The invariant of degree 1 is associated with the two-dimensional representation of $B_{2}$ and we set

$$
g_{0}(q)=2\left(q_{1}+q_{4}\right)
$$

There are 4 linearly independent invariants of degree 2 , the two-sided $\sum_{i=1}^{4} q_{i}^{2}$, and

$$
\begin{aligned}
& g_{1}(q)=q_{1} q_{4}+q_{2} q_{3} \\
& g_{2}(q)=\frac{1}{2}\left(q_{1}^{2}-q_{2}^{2}-q_{3}^{2}+q_{4}^{2}\right) \\
& g_{3}(q)=q_{1} q_{4}-q_{2} q_{3} .
\end{aligned}
$$

Then

$$
\begin{aligned}
& g_{1}\left(q \lambda\left(\sigma_{1}\right)\right)=-g_{1}(q), g_{2}\left(q \lambda\left(\sigma_{1}\right)\right)=g_{2}(q), g_{3}\left(q \lambda\left(\sigma_{1}\right)\right)=-g_{3}(q), \\
& g_{1}\left(q \lambda\left(\sigma_{2}\right)\right)=g_{1}(q), g_{2}\left(q \lambda\left(\sigma_{2}\right)\right)=-g_{2}(q), g_{3}\left(q \lambda\left(\sigma_{2}\right)\right)=-g_{3}(q) .
\end{aligned}
$$

Further $g_{i}(1,0,0,1)=1$ for $1 \leq i \leq 3$ (a plausible normalization). By simple orthogonality arguments we see that if $f(x)$ is a polynomial of even degree then $\int_{\Omega} f(x \tau(q)) g_{i}(q) d \mu(q) \neq 0$ only if $f(x)$ has a nonzero component of determinant type for $i=3$, or type $i$ for $i=1,2$. Experimentation quickly showed that the formula $V f(x)=\int_{\Omega} f(x \tau(q)) g_{3}(q) d \mu(q)$ appeared to be valid for the determinant type, but the similar attempt failed for types 1 and 2. (In fact, no polynomial in $q$ of degree less than twelve with the correct behavior under the $B_{2}$-action works.) We comment on this quandary in Section 4. We set

$$
\partial_{i}=\frac{\partial}{\partial q_{i}}, 1 \leq i \leq 4 .
$$

The formula for $V$ comes from applying the adjoint action $L:=\operatorname{ad}\left(\frac{1}{2} \sum_{i=1}^{4} \partial_{i}^{2}\right)$ to (multiplication by) the $g_{i}$. $(\operatorname{Recall}$ that $(\operatorname{ad}(A) B) f:=(A B-B A) f$ for operators $A, B$ on polynomials $f$.) Indeed

$$
\begin{aligned}
& L\left(g_{1}\right)=q_{1} \partial_{4}+q_{4} \partial_{1}+q_{2} \partial_{3}+q_{3} \partial_{2}, \\
& L\left(g_{2}\right)=q_{1} \partial_{1}-q_{2} \partial_{2}-q_{3} \partial_{3}+q_{4} \partial_{4}, \\
& L\left(g_{3}\right)=q_{1} \partial_{4}+q_{4} \partial_{1}-q_{2} \partial_{3}-q_{3} \partial_{2} .
\end{aligned}
$$

These operators have the same invariance properties as the respective polynomials $g_{i}$. The motivation for introducing the operator $L$ is to prove these invariance properties (in fact, the differential operators were found first by experimentation, and this explanation was noticed later). Set $D_{0}:=L\left(g_{1}+g_{2}\right)=\left(q_{1}+q_{4}\right)\left(\partial_{1}+\partial_{4}\right)-\left(q_{2}-q_{3}\right)\left(\partial_{2}-\partial_{3}\right)$ and $D_{3}:=L\left(g_{3}\right)$.

The formula stated in the introduction can be given as a pure integral with no derivatives for $\kappa>\frac{3}{2}$. However another variable of integration occurs (a total of seven!). 
Let $\omega$ denote the integration operator defined by

$$
\omega f(x):=\int_{0}^{1} f(t x) t^{4 \kappa-1} d t
$$

so that $\omega f(x)=\frac{1}{4 \kappa+n} f(x)$ when $f$ is homogeneous of degree $n$.

THEOREM 4. Let $\kappa>\frac{3}{2}$ and suppose that $f$ is sufficiently smooth on a ball $B_{R}:=$ $\left\{x \in \mathbb{R}^{2}:\|x\|_{2}:=\langle x, x\rangle^{1 / 2}<R\right\}$ for some $R>0$. For $x \in B_{R}$, we have

$$
\begin{aligned}
V f(x)= & \int_{\Omega} f(x \tau(q))\left(1+g_{0}(q)+g_{3}(q)\right) d \mu(q) \\
& +(2 \kappa-3) \int_{\Omega} \omega f(x \tau(q)) \widetilde{g}_{0}\left(q, u, \theta, \phi_{1}, \phi_{2}, \psi_{1}, \psi_{2}\right) d \mu(q)
\end{aligned}
$$

where

$$
\widetilde{g}_{0}=\frac{1}{1+\cos \theta}\left(\frac{q_{1}+q_{4}}{u \sin \phi_{1} \sin \psi_{1}}\right)^{2}-\frac{1}{1-\cos \theta}\left(\frac{q_{2}-q_{3}}{(1-u) \sin \phi_{2} \sin \psi_{2}}\right)^{2} .
$$

If $f(x)$ is homogeneous of degree $n$ then $\int_{\Omega} f(x \tau(q)) g_{3}(q) d \mu(q)$ is zero when $n$ is odd and equals $\frac{1}{8 \kappa+n} \int_{\Omega} D_{3} f(x \tau(q)) d \mu(q)$ when $n$ is even. (The proof is in the next subsection.) Also we shall prove the two equations (1.2) and (1.3). The factor $\frac{1}{4 \kappa+n}$ in (1.3) can be replaced by the integral operator $\omega$. The following result uses an integration by parts to replace the differential operator $D_{0}$ by an integral, the last ingredient of the formula in the theorem.

LEMMA 2. For $\kappa>\frac{3}{2}$ and a smooth function $h(q)$

$$
\begin{aligned}
\int_{\Omega} D_{0} h(q) d \mu(q)= & (2 \kappa-3) \int_{\Omega} h(q)\left\{\frac{1}{1+\cos \theta}\left(\frac{q_{1}+q_{4}}{u \sin \phi_{1} \sin \psi_{1}}\right)^{2}\right. \\
& \left.-\frac{1}{1-\cos \theta}\left(\frac{q_{2}-q_{3}}{(1-u) \sin \phi_{2} \sin \psi_{2}}\right)^{2}\right\} d \mu(q) .
\end{aligned}
$$

Proof. Use the notation and change-of-variable from Lemma 1 to set up the integration by parts. The measure is $d \mu=c_{\kappa}\left(z_{1} z_{2}\right)^{\kappa-3 / 2}(\sin \theta)^{2 \kappa-3} d q_{1} d q_{2} d q_{3} d q_{4} d u d \theta$ and the region of integration $\Omega_{1}$ is implicitly defined by $z_{1} \geq 0, z_{2} \geq 0,0 \leq \theta \leq \pi, 0 \leq u \leq 1$. If $w(q)$ vanishes on the boundary of the bounded domain $Q$ in $\mathbb{R}^{4}$ then $\int_{Q} D_{0} h(q) w(q) d q=$ $-\int_{Q} h(q) D_{0} w(q) d q$. (Although $\int_{Q} q_{j} \frac{\partial}{\partial q_{j}} h(q) w(q) d q=-\int_{Q} h(q)\left(1+q_{j} \frac{\partial}{\partial q_{j}}\right) w(q) d q$ for $1 \leq j \leq 4$ the terms $\int_{Q} h(q) w(q) d q$ cancel out.) We find that

$$
\begin{aligned}
& D_{0} z_{1}=-2 \frac{(1-\cos \theta)\left(q_{1}+q_{4}\right)^{2}}{\sin ^{2} \theta}=-2 \frac{\left(q_{1}+q_{4}\right)^{2}}{1+\cos \theta}, \\
& D_{0} z_{2}=2 \frac{(1+\cos \theta)\left(q_{2}-q_{3}\right)^{2}}{\sin ^{2} \theta}=2 \frac{\left(q_{2}-q_{3}\right)^{2}}{1-\cos \theta},
\end{aligned}
$$

and $D_{0}\left(z_{1} z_{2}\right)^{\kappa-3 / 2}=\left(\kappa-\frac{3}{2}\right)\left(\frac{D_{0} z_{1}}{z_{1}}+\frac{D_{0} z_{2}}{z_{2}}\right)\left(z_{1} z_{2}\right)^{\kappa-3 / 2}$. Then we change back to the original variables $\phi_{1}, \phi_{2}, \psi_{1}, \psi_{2}, u, \theta$ to complete the proof. 
2.2. Proof of the intertwining property. Suppose that $\xi$ is a linear functional on polynomials in $q=\left(q_{1}, q_{2}, q_{3}, q_{4}\right)$, and define an operator on polynomials in $x=$ $\left(x_{1}, x_{2}\right)$ by $V_{1} f(x)=\xi f\left(x_{1} q_{1}+x_{2} q_{2}, x_{1} q_{3}+x_{2} q_{4}\right)$. What needs to be done to show that $V_{1}=V$, the intertwining operator? The group invariance requires that $\xi p(q)=$ $\xi p\left(q_{4}, q_{3}, q_{2}, q_{1}\right)=\xi p\left(q_{1},-q_{2},-q_{3}, q_{4}\right)$.

Definition 2. Let $\xi_{0}$ denote the functional defined by $q^{\alpha} \mapsto s(\alpha)$. For a homogeneous polynomial $p(q)$ in $q$ of degree $n$ define the functional $\xi$ by:

$$
\begin{aligned}
& \xi(p)=\xi_{0}\left(g_{0} p\right), \quad \text { for } n \text { odd } \\
& \xi(p)=\xi_{0}\left(\left(1+\frac{1}{4 \kappa+n} D_{0}+\frac{1}{8 \kappa+n} D_{3}\right) p(q)\right), \quad \text { for } n \text { even. }
\end{aligned}
$$

When the criterion from [4, Prop. 1.3] for $V_{1}=V$ is expanded in homogeneous polynomials (that is, $\exp (\langle x, y\rangle)=\sum_{n=0}^{\infty} \frac{\langle x, y\rangle^{n}}{n !}$ ) one obtains:

$$
\begin{aligned}
& (n+1)\left(\langle x, y\rangle \xi\left(\langle x \tau(q), y\rangle^{n}\right)-\xi\left(\langle x \tau(q), y\rangle^{n+1}\right)\right) \\
& =\kappa \sum_{i=1}^{4}\left(\xi\left(\langle x \tau(q), y\rangle^{n+1}\right)-\xi\left(\left\langle x \sigma_{i} \tau(q), y\right\rangle^{n+1}\right)\right),
\end{aligned}
$$

for $n=0,1,2, \ldots$, where $\left\{\sigma_{i}\right\}$ is the set of reflections $\left\{\sigma_{1}, \sigma_{2}, \sigma_{2} \sigma_{1} \sigma_{2}, \sigma_{1} \sigma_{2} \sigma_{1}\right\}$, and $\sigma_{i} \tau(q)=\tau\left(q \lambda\left(\sigma_{i}\right)\right)$. Rewrite the criterion as follows:

$$
\begin{aligned}
& \xi\left(\left(\partial_{1}+\partial_{4}\right)\langle x \tau(q), y\rangle^{n+1}\right)-(n+1+4 \kappa) \xi\left(\langle x \tau(q), y\rangle^{n+1}\right) \\
& \quad+\kappa \sum_{i=1}^{4} \xi\left(\left\langle x \tau\left(q \lambda\left(\sigma_{i}\right)\right), y\right\rangle^{n+1}\right)=0 .
\end{aligned}
$$

Note that $\left(\partial_{1}+\partial_{4}\right)\langle x \tau(q), y\rangle=\langle x, y\rangle$. We shall prove that $\xi$ satisfies the criterion and also that $\xi_{0}\left(D_{3} p(q)\right)=(8 \kappa+n) \xi_{0}\left(g_{3} p\right)$ when $p$ is of degree $n$. (Both sides vanish when $n$ is odd.) We have

$$
\begin{aligned}
q \lambda\left(\sigma_{1}\right) & =\left(-q_{1}, q_{2},-q_{3}, q_{4}\right), \\
q \lambda\left(\sigma_{2}\right) & =\left(q_{2}, q_{1}, q_{4}, q_{3}\right), \\
q \lambda\left(\sigma_{1} \sigma_{2} \sigma_{1}\right) & =\left(-q_{2},-q_{1},-q_{4},-q_{3}\right), \\
q \lambda\left(\sigma_{2} \sigma_{1} \sigma_{2}\right) & =\left(q_{1},-q_{2}, q_{3},-q_{4}\right) .
\end{aligned}
$$

Then

$$
\begin{aligned}
& \sum_{i=1}^{4} g_{j}\left(q \lambda\left(\sigma_{i}\right)\right)=0, \quad \text { for } j=0,1,2 \\
& \sum_{i=1}^{4} g_{3}\left(q \lambda\left(\sigma_{i}\right)\right)=-4 g_{3}(q) .
\end{aligned}
$$

The corresponding differential operators $L\left(g_{j}\right)$ satisfy similar equations for $j=1,2,3$ because $\sum_{i=1}^{4} \partial_{i}^{2}$ is two-sided invariant; that is, $\sum_{i=1}^{4} L\left(g_{j}\right) p\left(q \lambda\left(\sigma_{i}\right)\right)=0$ for $j=1,2$ and $\sum_{i=1}^{4} L\left(g_{3}\right) p\left(q \lambda\left(\sigma_{i}\right)\right)=-4 L\left(g_{3}\right) p(q)$.

The proof of the criterion is easy when $n$ is odd. 

have

Proposition 3. Suppose that $n$ is odd and $p(q)$ is homogeneous of degree $n+1$. We

$$
\begin{aligned}
& \xi_{0}\left(g_{0}(q)\left(\partial_{1}+\partial_{4}\right) p(q)\right) \\
& \quad-(n+1+4 \kappa) \xi_{0}\left(\left(1+\frac{1}{4 \kappa+n+1} D_{0}+\frac{1}{8 \kappa+n+1} D_{3}\right) p(q)\right) \\
& \quad+\kappa \xi_{0}\left(\left(4-\frac{4}{8 \kappa+n+1} D_{3}\right) p(q)\right)=0 .
\end{aligned}
$$

Proof. In fact, the left side simplifies to

$$
\xi_{0}\left(\left(2\left(q_{1}+q_{4}\right)\left(\partial_{1}+\partial_{4}\right)-(n+1)-D_{0}-D_{3}\right) p(q)\right) .
$$

Replace $n+1$ by the Euler operator $\sum_{i=1}^{4} q_{i} \partial_{i}$. Then the expression becomes identically zero.

COROLlaRY 1. The criterion (2.1) is satisfied for odd $n$.

Proof. Set $p(q)=\langle x \tau(q), y\rangle^{n+1}$ in Proposition 3.

For the odd degree case ( $n$ even) replace $n$ by $2 n$ in the criterion. The third part vanishes because

$$
\begin{aligned}
\sum_{i=1}^{4} \xi\left(\left\langle x \tau\left(q \lambda\left(\sigma_{i}\right)\right), y\right\rangle^{2 n+1}\right) & =\sum_{i=1}^{4} \xi_{0}\left(g_{0}(q)\left\langle x \tau\left(q \lambda\left(\sigma_{i}\right)\right), y\right\rangle^{2 n+1}\right) \\
& =\sum_{i=1}^{4} \xi_{0}\left(g_{0}\left(q \lambda\left(\sigma_{i}\right)\right)\langle x \tau(q), y\rangle^{2 n+1}\right) \\
& =\xi_{0}\left(\sum_{i=1}^{4} g_{0}\left(q \lambda\left(\sigma_{i}\right)\right)\langle x \tau(q), y\rangle^{2 n+1}\right) \\
& =0 .
\end{aligned}
$$

This used the invariance property of $\xi_{0}$. Hence it is required to prove that

$$
(4 \kappa+2 n+1) \xi_{0}\left(\langle x \tau(q), y\rangle^{2 n+1} g_{0}(q)\right)-\xi\left(\left(\partial_{1}+\partial_{4}\right)\langle x \tau(q), y\rangle^{2 n+1}\right)=0 .
$$

This equation can be restated as follows:

$$
(4 \kappa+2 n+1) K_{2 n+1}(x, y)=\langle x, y\rangle K_{2 n}(x, y) .
$$

Recall the definition of $P_{a, b}^{c}(q)$ from (1.6). Thus

$$
\langle x \tau(q), y\rangle^{n}=\sum_{i=0}^{n}\left(\begin{array}{l}
n \\
i
\end{array}\right) y_{1}^{n-i} y_{2}^{i} \sum_{c=0}^{n} P_{n-i, i}^{c}(q) x_{1}^{n-c} x_{2}^{c},
$$

for $n \in \mathbb{N}_{0}$, so that it suffices to prove identities involving $\xi$ and $\langle x \tau(q), y\rangle^{n}$ for the polynomials $P_{a, b}^{c}$. There are two immediate consequences of the invariance properties of $\xi$. Since

$$
\begin{aligned}
P_{a, b}^{c}\left(q \lambda\left(\sigma_{1}\right) \rho\left(\sigma_{1}\right)\right) & =(-1)^{b+c} P_{a, b}^{c}(q), \\
\xi\left(P_{a, b}^{c}\right) & \neq 0 \Longrightarrow b \equiv c \bmod 2
\end{aligned}
$$


and since

$$
\begin{aligned}
P_{a, b}^{c}\left(q \lambda\left(\sigma_{2}\right) \rho\left(\sigma_{2}\right)\right) & =P_{b, a}^{a+b-c}(q), \\
\xi\left(P_{a, b}^{c}\right) & =\xi\left(P_{b, a}^{a+b-c}\right) .
\end{aligned}
$$

With the aim of applying condition (2.2) to $P_{a, b}^{c}$ with $a+b=2 n+1$ we can assume that $a$ is odd, $b$ and $c$ are even. This implies that $\xi_{0}\left(q_{4} P_{a, b}^{c}(q)\right)=0$. (The typical monomial is $q_{1}^{a-c+i} q_{2}^{c-i} q_{3}^{b-i} q_{4}^{i+1}$; the parities of the exponents of $q_{3}$ and $q_{4}$ are opposite.)

In the evaluation of $\xi_{0}\left(g_{0} P_{a, b}^{c}\right)$ and $\xi\left(\left(\partial_{1}+\partial_{4}\right) P_{a, b}^{c}\right)$ there are several vanishing terms:

$$
\begin{aligned}
\xi_{0}\left(q_{4} P_{a, b}^{c}\right) & =\xi_{0}\left(\partial_{4} P_{a, b}^{c}\right)=\xi_{0}\left(q_{i} \partial_{i} \partial_{4} P_{a, b}^{c}\right)=0, \quad \text { for } 1 \leq i \leq 4, \\
\xi_{0}\left(q_{4} \partial_{1}^{2} P_{a, b}^{c}\right) & =\xi_{0}\left(q_{1} \partial_{4} \partial_{1} P_{a, b}^{c}\right)=\xi_{0}\left(q_{2} \partial_{3} \partial_{1} P_{a, b}^{c}\right)=\xi_{0}\left(q_{3} \partial_{2} \partial_{1} P_{a, b}^{c}\right)=0 .
\end{aligned}
$$

Thus

$$
\begin{aligned}
& \xi_{0}\left(D_{0}\left(\partial_{1}+\partial_{4}\right) P_{a, b}^{c}\right)=\xi_{0}\left(L\left(g_{2}\right) \partial_{1} P_{a, b}^{c}\right)+\xi_{0}\left(L\left(g_{1}\right) \partial_{4} P_{a, b}^{c}\right) \\
& \xi_{0}\left(D_{3}\left(\partial_{1}+\partial_{4}\right) P_{a, b}^{c}\right)=\xi_{0}\left(D_{3} \partial_{4} P_{a, b}^{c}\right) .
\end{aligned}
$$

The required identity is

$$
\begin{aligned}
& (4 \kappa+2 n+1) \xi_{0}\left(2 q_{1} P_{a, b}^{c}\right)-\xi_{0}\left(\left(1+\frac{1}{4 \kappa+2 n} L\left(g_{2}\right)\right) \partial_{1} P_{a, b}^{c}\right) \\
& \quad-\frac{1}{(2 \kappa+n)(4 \kappa+n)} \xi_{0}\left(\left\{(3 \kappa+n)\left(q_{4} \partial_{1}+q_{1} \partial_{4}\right)+\kappa\left(q_{3} \partial_{2}+q_{2} \partial_{3}\right)\right\} \partial_{4} P_{a, b}^{c}\right) \\
& \quad=0 .
\end{aligned}
$$

We shall prove this by summing over the monomials in $P_{a, b}^{c}$; that is, we replace $P_{a, b}^{c}$ in the left side by $q_{1}^{a-c+i} q_{2}^{c-i} q_{3}^{b-i} q_{4}^{i}$ and evaluate $\xi_{0}$ (in terms of $s(\alpha)$ ). The partial sums are found explicitly by use of contiguity relations for the ${ }_{4} F_{3}$-type function $F$.

Set $a=2 a_{1}+1+2 a_{3}, b=2 a_{2}, c=2 a_{3}$ so that so $n=a_{1}+a_{2}+a_{3}$. Assume for now that $a_{1} \geq 0$; that is, $a>c$. Evaluate the left side for the monomial $q^{\alpha}$ with $\alpha=\left(2 a_{1}+1+i, 2 a_{3}-i, 2 a_{2}-i, i\right)$. (Note $|\alpha|=2 n+1$.) One of the terms simplifies:

$$
\begin{aligned}
\xi_{0} & \left(\left(1+\frac{1}{4 \kappa+2 n} L\left(g_{2}\right)\right) \partial_{1} q^{\alpha}\right) \\
& =\left(2 a_{1}+1+i\right)\left(1+\frac{2 a_{1}-2 a_{2}-2 a_{3}-4 i}{4 \kappa+2 n}\right) s\left(2 a_{1}+i, 2 a_{3}-i, 2 a_{2}-i, i\right) \\
& =2\left(2 a_{1}+1+i\right) \frac{\left(\kappa+a_{1}-i\right)}{2 \kappa+n} s\left(2 a_{1}+i, 2 a_{3}-i, 2 a_{2}-i, i\right) .
\end{aligned}
$$

To remove some common factors we divide by $s\left(2 a_{1}+2,2 a_{3}, 2 a_{2}, 0\right)$ (see Proposition 2 for the evaluation) and denote the result by $t_{i}$. The following expression 
is a linear combination of $F$ values with simple coefficients

$$
\begin{aligned}
s\left(2 a_{1}+2,2 a_{3}, 2 a_{2}, 0\right) t_{i}= & 2(4 \kappa+2 n+1) s\left(2 a_{1}+2+i, 2 a_{3}-i, 2 a_{2}-i, i\right) \\
& -2 \frac{\left(2 a_{1}+1+i\right)\left(\kappa+a_{1}+i\right)}{2 \kappa+n} s\left(2 a_{1}+i, 2 a_{3}-i, 2 a_{2}-i, i\right) \\
& -\frac{i(3 \kappa+n)}{(2 \kappa+n)(4 \kappa+n)}\left\{\left(2 a_{1}+1+i\right) s\left(2 a_{1}+i, 2 a_{3}-i, 2 a_{2}-i, i\right)\right. \\
& \left.+(i-1) s\left(2 a_{1}+2+i, 2 a_{3}-i, 2 a_{2}-i, i-2\right)\right\} \\
& -\frac{i \kappa}{(2 \kappa+n)(4 \kappa+n)}\left\{( 2 a _ { 3 } - i ) s \left(2 a_{1}+1+i, 2 a_{3}-i-1,2 a_{2}\right.\right. \\
& -i+1, i-1)+\left(2 a_{2}-i\right) s\left(2 a_{1}+1+i, 2 a_{3}\right. \\
& \left.\left.-i+1,2 a_{2}-i-1, i-1\right)\right\} .
\end{aligned}
$$

Then $t_{0}=0$ (directly). Rewrite $\left(\begin{array}{c}a \\ c-i\end{array}\right)\left(\begin{array}{l}b \\ i\end{array}\right)=\left(\begin{array}{c}a \\ c\end{array}\right) \frac{(-c)_{i}(-b)_{i}}{i !(a-c+1)_{i}}=\left(\begin{array}{c}a \\ c\end{array}\right) \frac{\left(-2 a_{3}\right)_{i}\left(-2 a_{2}\right)_{i}}{i !\left(2 a_{1}+2\right)_{i}}$.

The proof of the following result is in Section 3.3.

THEOREM 5. Suppose that $a_{1} \geq 0$ and $m \geq 1$. Then

$$
\begin{aligned}
& \sum_{i=1}^{m} \frac{\left(-2 a_{3}\right)_{i}\left(-2 a_{2}\right)_{i}}{i !\left(2 a_{1}+2\right)_{i}} t_{i}=\frac{2^{2 m+3} \kappa a_{2} a_{3}\left(2-2 a_{2}\right)_{m-1}\left(2-2 a_{3}\right)_{m-1}\left(\kappa+a_{1}+1\right)_{m}}{(m-1) !\left(-2 \kappa-2 a_{2}-2 a_{3}+1\right)_{2 m}} \\
& \quad \times \frac{\left(a_{1}+\frac{3}{2}\right)_{m-1}(4 \kappa+2 n+1)}{\left(2 a_{1}+2\right)_{m}(4 \kappa+n)} F\left(m-1 ; \kappa+1, a_{1}+1, a_{2}-1, a_{3}-1\right) .
\end{aligned}
$$

COROLlARY 2. The identity (2.3) is valid and criterion (2.1) is satisfied for even $n$.

Proof. The factor $\left(2-2 a_{2}\right)_{m-1}\left(2-2 a_{3}\right)_{m-1}$ vanishes for $m \geq 2 a_{2}$ or $m \geq 2 a_{3}$. Multiply both sides by $\left(\begin{array}{c}2 a_{1}+2 a_{3}+1 \\ 2 a_{3}\end{array}\right)$. The left side becomes

$$
\sum_{i=0}^{2 a_{3}} \frac{\left(-2 a_{2}\right)_{i}\left(-2 a_{1}-2 a_{3}-1\right)_{2 a_{3}-i}}{i\left(2 a_{3}-i\right)} t_{i} .
$$

If $a_{1} \geq 0$ set $m=\min \left(2 a_{2}, 2 a_{3}\right)$. The poles of $F$ (as a rational function of $\left.a_{2}, a_{3}\right)$ occur in a subset of $\frac{1}{2}+\mathbb{N}_{0}$. Next we use a weak form of analytic continuation to apply the Theorem to the case $a_{1}<0$. The terms in the left side with $2 a_{3}-i-1+\left(-2 a_{1}-2 a_{3}-\right.$ 1) $\geq 0$ vanish; that is, for $i<-2 a_{1}-1$. Further $\left(2 a_{1}+1\right) ! \times\left(2 a_{1}+2\right)_{m}=\left(2 a_{1}+m+\right.$ $1)$ !). Since $1 /\left(2 a_{1}+m+1\right)$ ! is entire for fixed $m$ the identity (left side minus right side, for fixed $\left.a_{2}, a_{3}, m\right)$ can be considered as a meromorphic function of $a_{1}$ vanishing for all $a_{1} \geq 0$ except possibly at the poles, which form a subset of $\frac{1}{2}+\mathbb{Z}$. Now let $a_{1} \rightarrow-\ell$ with $1 \leq \ell \leq a_{3}$. The terms in the left side (the sum) vanish for $i \leq 2 \ell-2$ and the right side vanishes for $2 a_{1}+m+1 \leq-1$; that is, $m \leq 2 \ell-2$. By analytic continuation $\sum_{i=2 \ell-1}^{\min \left(2 a_{2}, 2 a_{3}\right)}\left(\begin{array}{c}2 a_{1}+2 a_{3}+1 \\ 2 a_{3}-i\end{array}\right)\left(\begin{array}{c}2 a_{2} \\ i\end{array}\right) t_{i}=0$. It remains to change the normalizing factor in $t_{i}$ to $s\left(1,2 a_{3}+2 a_{1}+1,2 a_{2}+2 a_{1}+1,-2 a_{1}-1\right)$. Note that $0 \leq c \leq a+b$ implies $2 a_{1}+2 a_{2}+1 \geq 0$. The formal expression (put $i=-2 a_{1}-1=2 \ell-1$ )

$$
\begin{aligned}
& \frac{s\left(1,2 a_{3}+2 a_{1}+1,2 a_{2}+2 a_{1}+1,-2 a_{1}-1\right)}{s\left(2 a_{1}+2,2 a_{3}, 2 a_{2}, 0\right)} \\
& \quad=\frac{\left(\frac{1}{2}-\kappa-a_{2}\right)_{\ell-1}\left(\frac{1}{2}-\kappa-a_{3}\right)_{\ell-1}\left(\kappa+a_{1}+1\right)_{2 \ell-1}\left(\frac{3}{2}+a_{1}\right)_{2 \ell-1}}{\left(\frac{1}{2}-a_{2}\right)_{\ell-1}\left(\frac{1}{2}-a_{3}\right)_{\ell-1}\left(\frac{1}{2}-\kappa-a_{2}-a_{3}\right)_{2 \ell-1}\left(1-\kappa-a_{2}-a_{3}\right)_{2 \ell-1}}
\end{aligned}
$$


has no poles or zeros at integer values of $a_{1}$. Thus the identity remains valid when multiplied by this ratio.

The formula for the partial sum in the Theorem was discovered by experimentation, and recognizing that the factorization of the partial sum produces linear factors and an irreducible polynomial in $(\kappa+1)(\kappa+n)$. The validity is proved by induction and a contiguity relation for $F$; (that is, to show $\sum_{i=1}^{m} c_{i}=d_{m}$ for sequences $\left\{c_{i}\right\},\left\{d_{m}\right\}$ it suffices to show $d_{0}=0$ and $c_{m}+d_{m-1}-d_{m}=0$ for $m \geq 1$ ). We use a similar approach to the formula

$$
(8 \kappa+2 n) \xi_{0}\left(\left(q_{1} q_{4}-q_{2} q_{3}\right)\langle x \tau(q), y\rangle^{2 n}\right)-\xi_{0}\left(D_{3}\langle x \tau(q), y\rangle^{2 n}\right)=0
$$

for $n=1,2,3, \ldots$ The expression $(8 \kappa+2 n) \xi_{0}\left(\left(q_{1} q_{4}-q_{2} q_{3}\right) P_{a, b}^{c}(q)\right)-\xi_{0}\left(D_{3} P_{a, b}^{c}(q)\right)$ with $a+b=2 n$ vanishes when $a$ and $b$ are even, and so it suffices to take $a, b, c$ all odd. Furthermore assume $a \geq c$, otherwise use the symmetry $P_{a, b}^{c}\left(q_{4}, q_{3}, q_{2}, q_{1}\right)=$ $P_{b, a}^{a+b-c}(q)$, which produces the same integral because of the invariance properties of $q_{1} q_{4}-q_{2} q_{3}$ and $\mu$, and replace $a, b, c$ by $b, a, a+b-c$ respectively. Note that $a+b-c$ is odd and $c \geq a$ implies $a+b-c \leq b$. Let $a=2 a_{1}+2 a_{3}+1, b=2 a_{2}+1, c=2 a_{3}+$ 1 , so that $n=a_{1}+a_{2}+a_{3}+1$ and $a_{1}, a_{2}, a_{3} \geq 0$. The monomials in $P_{a, b}^{c}$ are $q^{\alpha}$ with $\alpha=\left(2 a_{1}+i, 2 a_{3}+1-i, 2 a_{2}+1-i, i\right)$ and $|\alpha|=2 n$. Set

$$
\begin{aligned}
& s\left(2 a_{1}, 2 a_{2}+2,2 a_{3}+2,0\right) t_{i} \\
&= \xi_{0}\left(\left((8 \kappa+2 n)\left(q_{1} q_{4}-q_{2} q_{3}\right)-D_{3}\right) q_{1}^{2 a_{1}+i} q_{2}^{2 a_{3}+1-i} q_{3}^{2 a_{2}+1-i} q_{4}^{i}\right) \\
&=(8 \kappa+2 n) s\left(2 a_{1}+1+i, 2 a_{3}+1-i, 2 a_{2}+1-i, i+1\right) \\
&-(8 \kappa+2 n) s\left(2 a_{1}+i, 2 a_{3}+2-i, 2 a_{2}+2-i, i\right) \\
&-\left(2 a_{1}+i\right) s\left(2 a_{1}+i-1,2 a_{3}+1-i, 2 a_{2}+1-i, i+1\right) \\
&-i s\left(2 a_{1}+i+1,2 a_{3}+1-i, 2 a_{2}+1-i, i-1\right) \\
&+\left(2 a_{3}+1-i\right) s\left(2 a_{1}+i, 2 a_{3}-i, 2 a_{2}+2-i, i\right) \\
&+\left(2 a_{2}+1-i\right) s\left(2 a_{1}+i, 2 a_{3}+2-i, 2 a_{2}-i, i\right) .
\end{aligned}
$$

The proof of the following result is in Section 3.

THEOREM 6. Suppose that $a_{1} \geq 0$ and $m=0,1,2, \ldots$ We have

$$
\begin{aligned}
\sum_{i=0}^{m} \frac{\left(-2 a_{2}-1\right)_{i}\left(-2 a_{3}-1\right)_{i}}{i !\left(2 a_{1}+1\right)_{i}} t_{i}= & \frac{2^{2 m+3} \kappa\left(\kappa+a_{1}\right)_{m+1}\left(-2 a_{2}\right)_{m}\left(-2 a_{3}\right)_{m}\left(a_{1}+\frac{1}{2}\right)_{m}}{m !\left(-2 \kappa-2 a_{2}-2 a_{3}-3\right)_{2 m+2}\left(2 a_{1}+1\right)_{m}} \\
& \times(4 \kappa+3 n+2) F\left(m ; \kappa+1, a_{1}, a_{2}, a_{3}\right) .
\end{aligned}
$$

COROllary 3. Equation (2.4) is valid.

Proof. Multiply the formula by $\left(\begin{array}{c}2 a_{1}+2 a_{3}+1 \\ 2 a_{3}+1\end{array}\right)$ and set $m=\min \left(2 a_{2}+1,2 a_{3}+1\right)$. The factor $\left(-2 a_{2}\right)_{m}\left(-2 a_{3}\right)_{m}$ vanishes for $m \geq 2 a_{2}+1$ or $m \geq 2 a_{3}+1$.

In the paper [4] the proof depended heavily on several integrations by parts. It may be possible that such a proof exists in this case (given sufficient ingenuity), but the method of integrating $P_{a, b}^{c}(q)$ by use of ${ }_{4} F_{3}$-series seems more straightforward. 


\section{Hypergeometric series tools.}

3.1. The single sum. A fundamental transformation for terminating ${ }_{3} F_{2}$ series (for $n \in \mathbb{N}_{0}$ ) is

$$
{ }_{3} F_{2}\left(\begin{array}{c}
-n, a, b \\
c, d
\end{array} ; 1\right)=\frac{(d-b)_{n}}{(d)_{n}}{ }_{3} F_{2}\left(\begin{array}{c}
-n, c-a, b \\
c, 1+b-d-n
\end{array} ; 1\right) .
$$

By iterating we obtain two useful transformations:

$$
{ }_{3} F_{2}\left(\begin{array}{c}
-n, a, b \\
c, d
\end{array} ; 1\right)=\frac{(c+d-a-b)_{n}}{(d)_{n}}{ }_{3} F_{2}\left(\begin{array}{l}
-n, c-a, c-b \\
c, c-a-b+d
\end{array} ; 1\right),
$$

and (this one provides the sum for the balanced case: $-n+a+b+1=c+d$ )

$$
\begin{aligned}
{ }_{3} F_{2}\left(\begin{array}{c}
-n, a, b \\
c, d
\end{array} d^{1}\right)= & (-1)^{n} \frac{(d-a)_{n}(d-b)_{n}}{(c)_{n}(d)_{n}} \\
& \times{ }_{3} F_{2}\left(\begin{array}{c}
-n, a+b-n+1-c-d, 1-d-n \\
a-d+1-n, b-d+1-n
\end{array} ; 1\right)
\end{aligned}
$$

We also need the Whipple transformation (see [1, p. 56]) for balanced terminating ${ }_{4} F_{3}$ series: if $n \in \mathbb{N}_{0}$ and $-n+a+b+c+1=c+d+e$, then

$$
\begin{aligned}
{ }_{4} F_{3}\left(\begin{array}{c}
-n, a, b, c \\
d, e, f
\end{array} ;\right)= & \frac{(e-a)_{n}(f-a)_{n}}{(e)_{n}(f)_{n}} \\
& \times{ }_{4} F_{3}\left(\begin{array}{c}
-n, a, d-b, d-c \\
d, e-b-c+d, f-b-c+d
\end{array} ; 1\right) .
\end{aligned}
$$

With the aid of these formulae we prove the single-sum expression for $s(\alpha)$. To concentrate on the intermediate steps we change some variables:

$$
\begin{aligned}
u_{1} & =\frac{1}{2}-b_{1}=\frac{1}{2}\left(1-\alpha_{1}-\alpha_{4}\right), \\
u_{2} & =\frac{1}{2}-b_{0}=\frac{1}{2}\left(1-\alpha_{2}-\alpha_{3}\right), \\
m & =\frac{1}{2}\left(\alpha_{3}-\alpha_{4}\right), \\
n & =\left\lfloor\frac{\alpha_{4}}{2}\right\rfloor .
\end{aligned}
$$

Also write $a$ for $\alpha_{4}$. By the parity condition $m$ is an integer; further the sets $\left\{\frac{a}{2}, \frac{a-1}{2}\right\}$ and $\left\{n, a-n-\frac{1}{2}\right\}$ are equal. For now we assume that $m>-n$ (this is necessary because factors $(m+1)_{i}$ with $0 \leq i \leq n$ will appear in denominators.) The special case $m=-n$, corresponding to $\alpha_{3}=0,1$ will be handled later. Start with the double sum from equation (1.4); note that $\left(-\alpha_{4}\right)_{2 i}=2^{2 i}\left(-\frac{\alpha_{4}}{2}\right)_{i}\left(\frac{1-\alpha_{4}}{2}\right)_{i}=2^{2 i}(-n)_{i}\left(n-a+\frac{1}{2}\right)_{i}$ 
and similarly $\left(-\alpha_{3}\right)_{2 j}=2^{2 j}(-m-n)_{j}\left(-m+n-a+\frac{1}{2}\right)_{j}$. Further we have $\frac{1}{2}-b_{3}=$ $\frac{1}{2}\left(1-\alpha_{3}-\alpha_{4}\right)=\frac{1}{2}-m-a$.

$$
\begin{aligned}
S & =\sum_{i=0}^{n} \sum_{j=0}^{m+n} \frac{(-n)_{i}\left(n-a+\frac{1}{2}\right)_{i}(-m-n)_{j}\left(-m+n-a+\frac{1}{2}\right)_{j}(\kappa)_{i+j}}{i ! j !\left(u_{1}\right)_{i}\left(u_{2}\right)_{j}\left(\frac{1}{2}-m-a\right)_{i+j}} \\
& \left.=\sum_{i=0}^{n} \frac{(-n)_{i}\left(n-a+\frac{1}{2}\right)_{i}(\kappa)_{i}}{i !\left(u_{1}\right)_{i}\left(\frac{1}{2}-m-a\right)_{i}}{ }_{3} F_{2}\left(\begin{array}{c}
-m-n,-m+n-a+\frac{1}{2}, \kappa+i \\
\frac{1}{2}-m-a+i, u_{2}
\end{array}\right]\right) \\
& =f_{1} \sum_{i=0}^{n} \frac{(-n)_{i}\left(n-a+\frac{1}{2}\right)_{i}(\kappa)_{i}}{i !\left(u_{1}\right)_{i}\left(\frac{1}{2}-m-a\right)_{i}}{ }_{3} F_{2}\left(\begin{array}{c}
-m-n, \frac{1}{2}-m-a-\kappa, i-n \\
\frac{1}{2}-m-a+i,-n-\kappa+u_{2}
\end{array} ; 1\right) \\
& =f_{1} \sum_{i=0}^{n} \sum_{j=0}^{n-i} \frac{(-n)_{i+j}\left(n-a+\frac{1}{2}\right)_{i}(-m-n)_{j}\left(\frac{1}{2}-m-a-\kappa\right)_{j}(\kappa)_{i}}{i ! j !\left(u_{1}\right)_{i}\left(-n-\kappa+u_{2}\right)_{j}\left(\frac{1}{2}-m-a\right)_{i+j}} \\
& =f_{1} S^{\prime}, f_{1}:=\frac{\left(-\kappa+u_{2}-n\right)_{n+m}}{\left(u_{2}\right)_{n+m}} .
\end{aligned}
$$

The transformation (3.1) is used on the inner sum. Note that the range of summation is now changed to $\{(i, j): i \geq 0, j \geq 0, i+j \leq n\}$, and $m$ can be considered a formal parameter. Reversing the order of summation and using equation (3.2) we obtain

$$
\begin{aligned}
S^{\prime}= & \sum_{j=0}^{n} \frac{(-n)_{j}(-m-n)_{j}\left(\frac{1}{2}-m-a-\kappa\right)_{j}}{j !\left(-n-\kappa+u_{2}\right)_{j}\left(\frac{1}{2}-m-a\right)_{j}}{ }_{3} F_{2}\left(\begin{array}{c}
j-n, n-a+\frac{1}{2}, \kappa \\
u_{1}, \frac{1}{2}-m-a+j
\end{array} ; 1\right) \\
= & \sum_{j=0}^{n} \frac{(-n)_{j}(-m-n)_{j}\left(\frac{1}{2}-m-a-\kappa\right)_{j}}{j !\left(-n-\kappa+u_{2}\right)_{j}\left(\frac{1}{2}-m-a\right)_{j}} \\
& \times(-1)^{n-j} \frac{(-m+j-n)_{n-j}\left(\frac{1}{2}-m-a-\kappa+j\right)_{n-j}}{\left(u_{1}\right)_{n-j}\left(\frac{1}{2}-m-a+j\right)_{n-j}} \\
& \times{ }_{3} F_{2}\left(\begin{array}{c}
j-n,-u_{1}+\kappa+m+1, \frac{1}{2}+m+a-n \\
\frac{1}{2}+\kappa+m+a-n, m+1
\end{array}\right),
\end{aligned}
$$

and reversing the order of summation again we obtain

$$
\begin{aligned}
S^{\prime}= & (-1)^{n} \frac{(-m-n)_{n}\left(\frac{1}{2}-m-a-\kappa\right)_{n}}{\left(\frac{1}{2}-m-a\right)_{n}\left(u_{1}\right)_{n}} \\
& \times \sum_{i=0}^{n} \sum_{j=0}^{n-i} \frac{(-n)_{i}\left(-u_{1}+\kappa+m+1\right)_{i}\left(\frac{1}{2}+m+a-n\right)_{i}(i-n)_{j}\left(1-n-u_{1}\right)_{j}}{i ! j !\left(\frac{1}{2}+\kappa+m+a-n\right)_{i}(m+1)_{i}\left(-n-\kappa+u_{2}\right)_{j}} \\
= & f_{2} \sum_{i=0}^{n} \frac{(-n)_{i}\left(-u_{1}+\kappa+m+1\right)_{i}\left(\frac{1}{2}+m+a-n\right)_{i}\left(u_{1}+u_{2}-\kappa-1\right)_{n-i}}{i !\left(\frac{1}{2}+\kappa+m+a-n\right)_{i}(m+1)_{i}\left(-n-\kappa+u_{2}\right)_{n-i}} \\
= & f_{2} S^{\prime \prime}, f_{2}:=\frac{(m+1)_{n}\left(\frac{1}{2}-m-a-\kappa\right)_{n}}{\left(\frac{1}{2}-m-a\right)_{n}\left(u_{1}\right)_{n}} .
\end{aligned}
$$


At the second last equation the $j$-sum is done with the Chu-Vandermonde sum $\left({ }_{2} F_{1}\left(\begin{array}{c}-n, b \\ c\end{array} ; 1\right)=\frac{(c-b)_{n}}{(c)_{n}}\right)$. Replace $i$ by $n-i$ in the sum $S^{\prime \prime}$, and use the relations $(b)_{n-i}=$ $(-1)^{i}(b)_{n} /(1-b-n)_{i}$ and $(-n)_{n-i} /(n-i) !=(-1)^{n}(-n)_{i} / i$ ! to obtain

$$
\begin{aligned}
S^{\prime \prime}= & (-1)^{n} \frac{\left(-u_{1}+\kappa+m+1\right)_{n}\left(\frac{1}{2}+m+a-n\right)_{n}}{\left(\frac{1}{2}+\kappa+m+a-n\right)_{n}(m+1)_{n}} \\
& \times{ }_{4} F_{3}\left(\begin{array}{c}
-n, u_{1}+u_{2}-\kappa-1, \frac{1}{2}-m-a-\kappa,-m-n \\
\frac{1}{2}-m-a, u_{1}-\kappa-m-n,-n-\kappa+u_{2}
\end{array} ; 1\right) \\
= & f_{3}{ }_{4} F_{3}\left(\begin{array}{c}
-n, u_{1}+u_{2}-\kappa-1, n-a+\frac{1}{2}, \kappa \\
\frac{1}{2}-m-a, u_{2}+m, u_{1}
\end{array}\right), \\
f_{3}= & (-1)^{n} \frac{\left(-u_{1}+\kappa+m+1\right)_{n}\left(\frac{1}{2}-m-a\right)_{n}\left(1-u_{1}-n\right)_{n}\left(1-u_{2}-m-n\right)_{n}}{\left(\frac{1}{2}-\kappa-m-a\right)_{n}(m+1)_{n}\left(-n-\kappa+u_{2}\right)_{n}\left(-n+u_{1}-\kappa-m\right)_{n}} .
\end{aligned}
$$

The Whipple transformation is valid since the first ${ }_{4} F_{3}$-series is balanced (as is the second, of course). We combine the factors

$$
\begin{aligned}
f_{1} f_{2} f_{3}= & \frac{\left(-\kappa+u_{2}-n\right)_{n+m}}{\left(u_{2}\right)_{n+m}} \frac{(m+1)_{n}\left(\frac{1}{2}-m-a-\kappa\right)_{n}}{\left(\frac{1}{2}-m-a\right)_{n}\left(u_{1}\right)_{n}} \\
& \times \frac{\left(-u_{1}+\kappa+m+1\right)_{n}\left(\frac{1}{2}-m-a\right)_{n}\left(u_{1}\right)_{n}\left(u_{2}+m\right)_{n}}{\left(\frac{1}{2}-\kappa-m-a\right)_{n}(m+1)_{n}\left(-n-\kappa+u_{2}\right)_{n}\left(-u_{1}+\kappa+m+1\right)_{n}} \\
= & \frac{\left(u_{2}-\kappa\right)_{m}}{\left(u_{2}\right)_{m}} .
\end{aligned}
$$

Thus

$$
S=\frac{\left(u_{2}-\kappa\right)_{m}}{\left(u_{2}\right)_{m}}{ }_{4} F_{3}\left(\begin{array}{c}
-n, u_{1}+u_{2}-\kappa-1, n-a+\frac{1}{2}, \kappa \\
\frac{1}{2}-m-a, u_{2}+m, u_{1}
\end{array} ;\right) .
$$

It is possible that $0>m>-n$ (that is, $\alpha_{3}<\alpha_{4}$ ) in which case the equation $(t)_{m}=$ $\frac{1}{(t+m)_{-m}}=\frac{(-1)^{m}}{(1-t)_{-m}}$ applies, and so $\frac{\left(u_{2}-\kappa\right)_{m}}{\left(u_{2}\right)_{m}}=\frac{\left(1-u_{2}\right)_{-m}}{\left(1+\kappa-u_{2}\right)_{-m}}$. Finally for the special case $m=-n$ the double sum $S$ reduces to the single sum

$$
\begin{aligned}
S & =\sum_{i=0}^{n} \frac{(-n)_{i}\left(n-a+\frac{1}{2}\right)_{i}(\kappa)_{i}}{i !\left(u_{1}\right)_{i}\left(\frac{1}{2}-m-a\right)_{i}}=\sum_{i=0}^{n} \frac{(-n)_{i}(\kappa)_{i}}{i !\left(u_{1}\right)_{i}} \\
& =\frac{\left(u_{1}-\kappa\right)_{n}}{\left(u_{1}\right)_{n}}
\end{aligned}
$$

while the ${ }_{4} F_{3}$ series reduces to a balanced ${ }_{3} F_{2}$ series, so that

$$
{ }_{3} F_{2}\left(\begin{array}{c}
-n, u_{1}+u_{2}-\kappa-1, \kappa \\
u_{2}-n, u_{1}
\end{array}\right)=(-1)^{n} \frac{\left(1+\kappa-u_{2}\right)_{n}\left(u_{1}-\kappa\right)_{n}}{\left(u_{2}-n\right)_{n}\left(u_{1}\right)_{n}},
$$

(by use of formula (3.2)) and the result multiplied by $\frac{\left(1-u_{2}\right)_{-m}}{\left(1+\kappa-u_{2}\right)_{-m}}$ produces $\frac{\left(u_{1}-\kappa\right)_{n}}{\left(u_{1}\right)_{n}}$, which equals $S$. 
This finishes the argument, and now we can show that the three-term recurrence applies to $s(\alpha)$. Returning to the original variables, we note that we have $b_{0}-m=\frac{1}{2}\left(\alpha_{2}+\alpha_{3}-\alpha_{3}+\alpha_{4}\right)=b_{2}$, and so

$$
\begin{aligned}
\frac{\left(u_{2}-\kappa\right)_{m}}{\left(u_{2}\right)_{m}} & =\frac{\left(\frac{1}{2}+b_{0}+\kappa-m\right)_{m}\left(\kappa+\frac{1}{2}\right)_{b_{2}}\left(\frac{1}{2}\right)_{b_{2}}}{\left(\frac{1}{2}+b_{0}-m\right)_{m}\left(\frac{1}{2}\right)_{b_{2}}\left(\kappa+\frac{1}{2}\right)_{b_{2}}} \\
& =\frac{\left(\kappa+\frac{1}{2}\right)_{b_{0}}\left(\frac{1}{2}\right)_{b_{2}}}{\left(\frac{1}{2}\right)_{b_{0}}\left(\kappa+\frac{1}{2}\right)_{b_{2}}} .
\end{aligned}
$$

This proves formula (1.7).

\subsection{Three term recurrence and symmetries.}

Proposition 4. For $n \in \mathbb{N}_{0}$

$$
\begin{aligned}
n(n+ & \left.2 v_{1}\right)\left(n+\kappa+v_{1}-\frac{1}{2}\right)\left(\kappa+\frac{1}{2}+v_{2}+v_{3}-n\right) F\left(n-1 ; \kappa, v_{1}, v_{2}, v_{3}\right) \\
& +\left\{n\left(n+2 v_{1}\right)\left(n-v_{2}-v_{3}-\frac{1}{2}\right)+\left(n+\frac{1}{2}+v_{1}\right)\left(n-2 v_{2}\right)\left(n-2 v_{3}\right)\right\} \\
& \times\left(n+v_{1}-\frac{1}{2}\right) F\left(n ; \kappa, v_{1}, v_{2}, v_{3}\right) \\
= & \left(n+v_{1}-\frac{1}{2}\right)\left(n+v_{1}+\frac{1}{2}\right)\left(n-2 v_{2}\right)\left(n-2 v_{3}\right) F\left(n+1 ; \kappa, v_{1}, v_{2}, v_{3}\right) .
\end{aligned}
$$

Proof. Expand the left side as a series in $g_{i}:=(\kappa)_{i}\left(-\kappa-v_{1}-v_{2}-v_{3}\right)_{i}$ for $0 \leq i \leq$ $\left\lfloor\frac{n+1}{2}\right\rfloor$. Start with the equation

$$
\begin{aligned}
& \left(n+\kappa+v_{1}-\frac{1}{2}\right)\left(\kappa+\frac{1}{2}+v_{2}+v_{3}-n\right) \\
& \quad=\left(\frac{1}{2}-v_{1}-n+i\right)\left(n-v_{2}-v_{3}-\frac{1}{2}+i\right)-(\kappa+i)\left(-\kappa-v_{1}-v_{2}-v_{3}+i\right) .
\end{aligned}
$$

The outline of the calculation is this: write $F(n-1)=\sum_{i \geq 0} a_{i} g_{i}$ and $F(n)=$ $\sum_{i \geq 0} a_{i}^{\prime} g_{i}$ (suppressing the other arguments); note that

$$
g_{i}(\kappa+i)\left(-\kappa-v_{1}-v_{2}-v_{3}+i\right)=g_{i+1} .
$$

Then collect term-by-term in

$$
\begin{aligned}
& \left(n+2 v_{1}\right)\left\{n\left(\frac{1}{2}-v_{1}-n+i\right)\left(n-v_{2}-v_{3}-\frac{1}{2}+i\right) \sum_{i \geq 0} a_{i} g_{i}\right. \\
& \left.-n \sum_{i \geq 1} a_{i-1} g_{i}+n\left(n-v_{2}-v_{3}-\frac{1}{2}\right)\left(n+v_{1}-\frac{1}{2}\right) \sum_{i \geq 0} a_{i}^{\prime} g_{i}\right\} \\
& +\left(n+\frac{1}{2}+v_{1}\right)\left(n+v_{1}-\frac{1}{2}\right)\left(n-2 v_{2}\right)\left(n-2 v_{3}\right) \sum_{i \geq 0} a_{i}^{\prime} g_{i}
\end{aligned}
$$


Write this sum as $\sum_{i \geq 0} \frac{\left(-\frac{n}{2}\right)_{i}\left(-\frac{1+n}{2}\right)_{i}}{i !\left(\frac{1}{2}-n-v_{1}\right)_{i}\left(\frac{1}{2}-v_{2}\right)_{i}\left(\frac{1}{2}-v_{3}\right)_{i}} c_{i} g_{i}$ and then (in corresponding order) we obtain

$$
\begin{aligned}
c_{i}= & \frac{n+2 v_{1}}{n+1}\left(n+v_{1}-\frac{1}{2}\right)\left\{-(n-2 i)(n+1-2 i)\left(n-v_{2}-v_{3}-\frac{1}{2}+i\right)\right. \\
& \left.+n(n+1-2 i)\left(n-v_{2}-v_{3}-\frac{1}{2}\right)+i\left(2 v_{2}+1-2 i\right)\left(2 v_{3}+1-i\right)\right\} \\
& +\frac{n+1-2 i}{n+1}\left(n+v_{1}-\frac{1}{2}\right)\left(n-2 v_{2}\right)\left(n-2 v_{3}\right) \\
= & \frac{\left(n-2 v_{2}\right)\left(n-2 v_{3}\right)}{n+1}\left(n+v_{1}-\frac{1}{2}\right)\left\{i\left(n+2 v_{1}\right)+\left(n+v_{1}+\frac{1}{2}\right)(n+1-2 i)\right\} \\
= & \left(n-2 v_{2}\right)\left(n-2 v_{3}\right)\left(n+v_{1}-\frac{1}{2}\right)\left(n+v_{1}+\frac{1}{2}+i\right) .
\end{aligned}
$$

Finally $\left(n+v_{1}+\frac{1}{2}+i\right) /\left(\frac{1}{2}-n-v_{1}\right)_{i}=\left(n+v_{1}+\frac{1}{2}\right) /\left(-\frac{1}{2}-n-v_{1}\right)_{i}$, which proves the identity.

COROLlaRY 4. The recurrence (1.5) for $s^{\prime}(\alpha)$ is valid.

Proof. Suppose that $\alpha_{1} \equiv \alpha_{2} \equiv \alpha_{3} \equiv \alpha_{4} \bmod 2$ and then set $c^{\prime}(\alpha)=\prod_{i=1}^{3} \frac{\left(\frac{1}{2}\right)_{b_{i}}}{\left(\kappa+\frac{1}{2}\right)_{b_{i}}}$ where $b_{i}=\frac{1}{2}\left(\alpha_{i}+\alpha_{4}\right), 1 \leq i \leq 3$. Then $s^{\prime}(\alpha) / c^{\prime}(\alpha)$ satisfies the recurrence for $F$ in the Proposition, with $n=\alpha_{4}, v_{1}=\frac{1}{2}\left(\alpha_{1}-\alpha_{4}\right), v_{2}=b_{2}, v_{3}=b_{3}$. Multiply the recurrence by $c^{\prime}(\alpha)$. Then the coefficient of $F(n-1)$ is multiplied by

$$
\frac{c^{\prime}(\alpha)}{c^{\prime}\left(\alpha_{1}-1, \alpha_{2}+1, \alpha_{3}+1, \alpha_{4}-1\right)}=\frac{\alpha_{1}+\alpha_{4}-1}{2 \kappa+\alpha_{1}+\alpha_{4}-1}
$$

and the coefficient of $F(n+1)$ is multiplied by

$$
\frac{c^{\prime}(\alpha)}{c^{\prime}\left(\alpha_{1}+1, \alpha_{2}-1, \alpha_{3}-1, \alpha_{4}+1\right)}=\frac{2 \kappa+\alpha_{1}+\alpha_{4}+1}{\alpha_{1}+\alpha_{4}+1} .
$$

Divide out the common factor $\frac{1}{2}\left(\alpha_{1}+\alpha_{4}-1\right)=\left(n+v_{1}-\frac{1}{2}\right)$ to obtain (1.5).

There is another, perhaps unexpected, symmetry.

Proposition 5. $s^{\prime}$ is completely symmetric in its arguments.

Proof. Already formula 1.7 shows the symmetry in $\left(\alpha_{1}, \alpha_{2}, \alpha_{3}\right)$. One can argue from the $\alpha_{2} \leftrightarrow \alpha_{3}$ and $\alpha_{1} \leftrightarrow \alpha_{4}$ invariance together with the $\alpha \leftrightarrow\left(\alpha_{2}, \alpha_{1}, \alpha_{4}, \alpha_{3}\right)$ invariance of $s$. The Whipple transformation (3.3) gives a direct proof. It suffices to show that $s^{\prime}\left(\alpha_{1}, \alpha_{2}, \alpha_{3}, \alpha_{4}\right)=s^{\prime}\left(\alpha_{4}, \alpha_{2}, \alpha_{3}, \alpha_{1}\right)$. If all the $\alpha_{i}$ 's are even take $n=\frac{\alpha_{4}}{2}, a=\kappa$ and $d=\frac{1}{2}-b_{1}$. Observe that

$$
\begin{aligned}
s^{\prime}\left(\alpha_{1}, \alpha_{2}, \alpha_{3}, \alpha_{4}\right)= & \frac{\left(\frac{1}{2}\right)_{\alpha_{2} / 2}\left(\frac{1}{2}\right)_{\alpha_{3} / 2}}{\left(\kappa+\frac{1}{2}\right)_{\alpha_{2} / 2}\left(\kappa+\frac{1}{2}\right)_{\alpha_{3} / 2}} \\
& \times{ }_{4} F_{3}\left(\begin{array}{c}
-\frac{\alpha_{4}}{2}, \kappa, \frac{-\alpha_{1}}{2}, \kappa+\frac{1}{2}\left(1+\alpha_{2}+\alpha_{3}\right) ; 1 \\
\frac{1}{2}-b_{1}, \kappa+\frac{1}{2}\left(1+\alpha_{3}\right), \kappa+\frac{1}{2}\left(1+\alpha_{2}\right)
\end{array} ; 1\right) .
\end{aligned}
$$


Note that this expression is symmetric in $\left(\alpha_{1}, \alpha_{4}\right)$. A similar argument works when $\alpha_{4}$ is odd:

$$
\begin{aligned}
s^{\prime}\left(\alpha_{1}, \alpha_{2}, \alpha_{3}, \alpha_{4}\right)= & \frac{\left(\frac{1}{2}\right)_{\left(\alpha_{2}+1\right) / 2}\left(\frac{1}{2}\right)_{\left(\alpha_{3}+1\right) / 2}}{\left(\kappa+\frac{1}{2}\right)_{\left(\alpha_{2}+1\right) / 2}\left(\kappa+\frac{1 x}{2}\right)_{\left(\alpha_{3}+1\right) / 2}} \\
& \times{ }_{4} F_{3}\left(\begin{array}{l}
\frac{1-\alpha_{4}}{2}, \kappa, \frac{1-\alpha_{1}}{2}, \kappa+\frac{1}{2}\left(1+\alpha_{2}+\alpha_{3}\right) \\
\frac{1}{2}-b_{1}, \kappa+1+\frac{\alpha_{3}}{2}, \kappa+1+\frac{\alpha_{2}}{2}
\end{array} ; 1\right) .
\end{aligned}
$$

3.3. Contiguity relations. We start by writing the expressions in Theorems 5 and 6 in terms of the function $F$ (see Definition 1). Suppose that $\alpha_{1} \equiv \alpha_{2} \equiv \alpha_{3} \equiv \alpha_{4} \bmod 2$ and then set

$$
c(\alpha)=\frac{(2 \kappa)_{\alpha_{1}+\alpha_{4}}(2 \kappa)_{\alpha_{2}+\alpha_{3}}}{(4 \kappa)_{|\alpha|}} \prod_{i=1}^{3} \frac{\left(\frac{1}{2}\right)_{b_{i}}}{\left(\kappa+\frac{1}{2}\right)_{b_{i}}}
$$

thus $s(\alpha)=c(\alpha) F\left(\alpha_{4} ; \kappa, \frac{1}{2}\left(\alpha_{1}-\alpha_{4}\right), b_{2}, b_{3}\right)$. For Theorem 5 we set $i=m \geq 1$ and extract the common factor

$$
\begin{aligned}
C_{m} & =\frac{c\left(2 a_{1}+m, 2 a_{3}-m, 2 a_{3}-m, m\right)\left(-2 a_{3}\right)_{m}\left(-2 a_{2}\right)_{m}}{s\left(2 a_{1}+2,2 a_{3}, 2 a_{2}, 0\right) m !\left(2 a_{1}+2\right)_{m}(4 \kappa+n)(2 \kappa+n)} \\
& =2^{2 m-1} \frac{(4 \kappa+2 n+1)\left(\kappa+a_{1}+1\right)_{m-1}\left(a_{1}+\frac{3}{2}\right)_{m-1}\left(-2 a_{3}\right)_{m}\left(-2 a_{2}\right)_{m}}{(4 \kappa+n)\left(1-2 \kappa-2 a_{2}-2 a_{3}\right)_{2 m} m !\left(2 a_{1}+2\right)_{m}}
\end{aligned}
$$

Recall that $n=\sum_{i=1}^{3} a_{i}$. When $i=0$ we have

$$
\begin{aligned}
t_{0} & =2(4 \kappa+2 n+1)-2 \frac{\left(2 a_{1}+1\right)\left(\kappa+a_{1}\right) c\left(2 a_{1}, 2 a_{3}, 2 a_{3}, 0\right)}{(2 \kappa+n) s\left(2 a_{1}+2,2 a_{3}, 2 a_{2}, 0\right)} \\
& =0 .
\end{aligned}
$$

The plan is to use induction by showing that

$$
\frac{\left(-2 a_{3}\right)_{m}\left(-2 a_{2}\right)_{m}}{m !\left(2 a_{1}+2\right)_{m}} t_{m}+d_{m-1}-d_{m}=0,
$$

where $d_{m}$ denotes the claimed expression for the sum. The following multipliers are needed in the calculation of $t_{m}$ :

$$
\begin{gathered}
\frac{c\left(2 a_{1}+m+2,2 a_{3}-m, 2 a_{2}-m, m\right)}{c\left(2 a_{1}+m, 2 a_{3}-m, 2 a_{2}-m, m\right)}=\frac{\left(\kappa+a_{1}+m\right)\left(2 a_{1}+2 m+1\right)}{(4 \kappa+2 n+1)(2 \kappa+n)}, \\
\frac{c\left(2 a_{1}+m+2,2 a_{3}-m, 2 a_{2}-m, m-2\right)}{c\left(2 a_{1}+m, 2 a_{3}-m, 2 a_{2}-m, m\right)}=\frac{\left(2 \kappa+2 a_{2}-1\right)\left(2 \kappa+2 a_{3}-1\right)}{\left(2 a_{2}-1\right)\left(2 a_{3}-1\right)}, \\
\frac{c\left(2 a_{1}+m+1,2 a_{3}-m-1,2 a_{2}-m+1, m-1\right)}{c\left(2 a_{1}+m, 2 a_{3}-m, 2 a_{2}-m, m\right)}=\frac{2 \kappa+2 a_{3}-1}{2 a_{3}-1}, \\
\frac{c\left(2 a_{1}+m+1,2 a_{3}-m+1,2 a_{2}-m-1, m-1\right)}{c\left(2 a_{1}+m, 2 a_{3}-m, 2 a_{2}-m, m\right)}=\frac{2 \kappa+2 a_{2}-1}{2 a_{2}-1} .
\end{gathered}
$$


Write $d_{m}=A_{m} F\left(m-1 ; \kappa+1, a_{1}+1, a_{2}-1, a_{3}-1\right)$. Then

$$
\begin{aligned}
\frac{A_{m}}{C_{m}} & =4 m \kappa\left(\kappa+a_{1}+m\right) \frac{\left(2 a_{2}-m\right)\left(2 a_{3}-m\right)}{\left(2 a_{2}-1\right)\left(2 a_{3}-1\right)}, \\
\frac{A_{m-1}}{A_{m}} & =\frac{2(m-1)\left(2 a_{1}+m+1\right)\left(\kappa+a_{2}+a_{3}-m\right)\left(\kappa+a_{2}+a_{3}+\frac{1}{2}-m\right)}{\left(\kappa+a_{1}+m\right)\left(2 a_{1}+2 m-1\right)\left(2 a_{2}-m\right)\left(2 a_{3}-m\right)} .
\end{aligned}
$$

The expression $\left(\frac{\left(-2 a_{3}\right)_{m}\left(-2 a_{2}\right)_{m}}{m !\left(2 a_{1}+2\right)_{m}} t_{m}+d_{m-1}-d_{m}\right) / C_{m}$ with each $a_{i}$ replaced by $v_{i}$ (formal parameters instead of integers) becomes the left side of the equation in the following result.

THEOREM 7. Suppose that $v_{1}, v_{2}, v_{3} \notin-\frac{1}{2}+\mathbb{N}_{0},\left(v_{0}=v_{1}+v_{2}+v_{3}\right)$ and $m=$ $1,2,3, \ldots$ Then

$$
\begin{aligned}
& 2\left(2 v_{1}+2 m+1\right)\left(\kappa+v_{1}+m\right)\left(4 \kappa+v_{0}\right) F\left(m ; \kappa, v_{1}+1, v_{2}, v_{3}\right) \\
& \quad-\left\{2\left(\kappa+v_{1}+m\right)\left(4 \kappa+v_{0}\right)+m\left(3 \kappa+v_{0}\right)\right\}\left(2 v_{1}+m+1\right) F\left(m ; \kappa, v_{1}, v_{2}, v_{3}\right) \\
& -m(m-1) \frac{\left(2 v_{2}+2 \kappa-1\right)\left(2 v_{3}+2 \kappa-1\right)}{\left(2 v_{2}-1\right)\left(2 v_{3}-1\right)}\left(3 \kappa+v_{0}\right) F\left(m-2 ; \kappa, v_{1}+2, v_{2}-1, v_{3}-1\right) \\
& \quad-m \kappa \frac{\left(2 \kappa+2 v_{3}-1\right)}{2 v_{3}-1}\left(2 v_{3}-m\right) F\left(m-1 ; \kappa, v_{1}+1, v_{2}, v_{3}-1\right) \\
& -m \kappa \frac{\left(2 \kappa+2 v_{2}-1\right)}{2 v_{2}-1}\left(2 v_{2}-m\right) F\left(m-1 ; \kappa, v_{1}+1, v_{2}-1, v_{3}\right) \\
& +m(m-1) \frac{8 \kappa\left(2 v_{1}+m+1\right)}{\left(2 v_{2}-1\right)\left(2 v_{3}-1\right)\left(2 v_{1}+2 m-1\right)}\left(\kappa+v_{2}+v_{3}-m\right) \\
& \quad \times\left(\kappa+v_{2}+v_{3}+\frac{1}{2}-m\right) F\left(m-2 ; \kappa+1, v_{1}+1, v_{2}-1, v_{3}-1\right) \\
& \quad-4 m \kappa \frac{\left(2 v_{2}-m\right)\left(2 v_{3}-m\right)}{\left(2 v_{2}-1\right)\left(2 v_{3}-1\right)}\left(\kappa+v_{1}+m\right) F\left(m-1 ; \kappa+1, v_{1}+1, v_{2}-1, v_{3}-1\right) \\
& =0 .
\end{aligned}
$$

Proof. We proceed by considering the left side as a polynomial in $\kappa$ of the form

$$
\sum_{i \geq 0}\left(c_{i}+d_{i} \kappa\right)(\kappa)_{i}\left(-\kappa-v_{1}-v_{2}-v_{3}\right)_{i} .
$$

The coefficients are rational functions of $v_{1}, v_{2}, v_{3}$ and $m$. Write the identity in abbreviated form as $\sum_{n=1}^{7} \mu_{n} F\left(\gamma_{n}\right)=0$ using the same order as above (for example, $\gamma_{2}=\left(m ; \kappa, v_{1}, v_{2}, v_{3}\right)$ and $\left.\mu_{5}=-m \kappa \frac{\left(2 \kappa+2 v_{2}-1\right)}{2 v_{2}-1}\left(2 v_{2}-m\right)\right)$. Denote term \#i of $F\left(\gamma_{n}\right)$ by $t_{i}\left(\gamma_{n}\right),\left(\right.$ where $t_{0}\left(\gamma_{n}\right)=1$ for all $\left.n\right)$, so that

$$
t_{i}\left(\gamma_{3}\right)=\frac{\left(1-\frac{m}{2}\right)_{i}\left(\frac{3-m}{2}\right)_{i}(\kappa)_{i}\left(-\kappa-v_{1}-v_{2}-v_{3}\right)_{i}}{\left(\frac{1}{2}-v_{1}-m\right)_{i}\left(\frac{3}{2}-v_{2}\right)_{i}\left(\frac{3}{2}-v_{3}\right)_{i} i !},
$$

for example, with the understanding that $F(m)=0$ for $m<0$. The verification is carried out by symbolic computation. The underlying idea is to set up the equations as rational functions of all of the variables. For example, $(a)_{i}$ is not rational in $i$, but for a fixed integer $k$ the expression $(a)_{i} /(a+k)_{i}$ is rational in $a$ and $i$ because of the identity 
$(a)_{i} /(a+k)_{i}=(a)_{k} /(a+i)_{k}$. By use of symbolic calculation we evaluate

$$
\begin{aligned}
& p_{i}(\kappa)=\mu_{1} \frac{t_{i+1}\left(\gamma_{1}\right)}{t_{i}\left(\gamma_{2}\right)}+\mu_{2}+\sum_{n=3}^{7} \mu_{n} \frac{t_{i}\left(\gamma_{n}\right)}{t_{i}\left(\gamma_{2}\right)}, \\
& p_{0}(\kappa)=\mu_{1}\left(1+t_{1}\left(\gamma_{1}\right)\right)+\mu_{2}+\sum_{n=3}^{7} \mu_{n},
\end{aligned}
$$

for $1 \leq i \leq\left\lfloor\frac{m}{2}\right\rfloor$. Indeed $p_{i}(\kappa)$ is a polynomial in $\kappa$ of degree 4 . (Note that $\frac{t_{i}\left(\gamma_{1}\right)}{t_{i}\left(\gamma_{2}\right)}$ is not a polynomial in $\kappa$ for $i \geq 1$, and the factor $\kappa$ in $\mu_{6}$ and $\mu_{7}$ is necessary since $\frac{t_{i}\left(\gamma_{n}\right)}{t_{i}\left(\gamma_{2}\right)}$ has $\kappa$ in the denominator for $n=6,7$.) Further $p_{i}(\kappa)$ is rational in all the variables $\left(v_{1}, v_{2}, v_{3}, \kappa, i, m\right)$. Although $i$ is an indexing variable the difference between the respective parameters in $\left\{\gamma_{n}\right\}$ is one of $0, \pm 1, \pm 2$ and thus $\frac{t_{i}\left(\gamma_{n}\right)}{t_{i}\left(\gamma_{2}\right)}$ is rational in $i$ for each $n$. By computer algebra we find the coefficients $\left\{c_{i, k}\right\}_{k=0}^{4}$, which are functions of $i, m, v_{1}, v_{2}, v_{3}$, so that

$$
p_{i}(\kappa)=c_{i, 0}+c_{i, 1} \kappa+\left(c_{i, 2}+c_{i, 3} \kappa\right) g_{i, 1}(\kappa)+c_{i, 4} g_{i, 2}(\kappa),
$$

where $g_{i, k}(\kappa):=(\kappa+i)_{k}\left(-\kappa-v_{0}+i\right)_{k}$ for $k \geq 0$. Thus $g_{0, i}(\kappa) g_{i, k}(\kappa)=g_{0, i+k}(\kappa)$. For example

$$
c_{i, 4}=\frac{4(2 i-m)(2 i+1-m)}{(i+1)\left(\frac{1}{2}-v_{2}+i\right)\left(\frac{1}{2}-v_{3}+i\right)}
$$

for $0 \leq i \leq\left\lfloor\frac{m}{2}\right\rfloor$, but the other coefficients are more complicated. Because the degree of $\kappa$ in $\mu_{1}$ is 2 the value of $c_{0,4}$ agrees with the generic $c_{i, 4}$ with $i=0$. For $i \geq 0$ let

$$
r_{i}=\frac{t_{i}\left(\gamma_{2}\right)}{g_{0, i}(\kappa)},
$$

so that $r_{i}$ is independent of $\kappa$ (thus $\left.r_{i}=\frac{\left(-\frac{m}{2}\right)_{i}\left(\frac{1-m}{2}\right)_{i}}{i !\left(\frac{1}{2}-v_{1}-m\right)_{i}\left(\frac{1}{2}-v_{2}\right)_{i}\left(\frac{1}{2}-v_{3}\right)_{i}}\right)$. The left side of the identity equals

$$
\begin{aligned}
& \sum_{i=0}^{\lfloor m / 2\rfloor} p_{i}(\kappa) g_{0, i}(\kappa) r_{i} \\
& \quad=\sum_{i=0}^{\lfloor m / 2\rfloor} r_{i}\left(\left(c_{i, 0}+c_{i, 1} \kappa\right) g_{0, i}+\left(c_{i, 2}+c_{i, 3} \kappa\right) g_{0, i+1}(\kappa)+c_{i, 4} g_{0, i+2}(\kappa)\right) \\
& \quad=\sum_{i=0}^{\lfloor m / 2\rfloor+2} g_{0, i}\left(r_{i}\left(c_{i, 0}+c_{i, 1} \kappa\right)+r_{i-1}\left(c_{i-1,2}+c_{i-1,3} \kappa\right)+r_{i-2} c_{i-2,4}\right) .
\end{aligned}
$$

By symbolic computation

$$
\left(c_{i, 0}+c_{i, 1} \kappa\right)+\frac{r_{i-1}}{r_{i}}\left(c_{i-1,2}+c_{i-1,3} \kappa\right)+\frac{r_{i-2}}{r_{i}} c_{i-2,4}=0
$$

for $2 \leq i \leq\left\lfloor\frac{m}{2}\right\rfloor$. Also

$$
\begin{aligned}
c_{0,0}+c_{0,1} \kappa & =0, \\
r_{1}\left(c_{1,0}+c_{1,1} \kappa\right)+r_{0}\left(c_{0,2}+c_{0,3} \kappa\right) & =0 .
\end{aligned}
$$


The special cases at the top end of summation occur at $i=\frac{m}{2}$ or $i=\frac{m-1}{2}$ (for $m$ being even or odd, respectively) and in fact $c_{i, 4}=0$ and $\left(c_{i, 2}+c_{i, 3} \kappa\right)+\frac{r_{i-1}}{r_{i}} c_{i-1,4}=0$ for these values of $i$.

This completes the proof of Theorem 5 .

We use the same approach to Theorem 6 , where we set $i=m \geq 0$ and extract the common factor

$$
\begin{aligned}
C_{m} & =\frac{c\left(2 a_{1}+m-1,2 a_{3}-m+1,2 a_{3}-m+1, m+1\right)\left(-2 a_{3}-1\right)_{m}\left(-2 a_{2}-1\right)_{m}}{s\left(2 a_{1}, 2 a_{3}+2,2 a_{2}+2,0\right) m !\left(2 a_{1}+1\right)_{m}(4 \kappa+2 n+1)(2 \kappa+n)} \\
& =2^{2 m+1} \frac{\left(\kappa+a_{1}\right)_{m}\left(a_{1}+\frac{1}{2}\right)_{m}\left(-2 a_{3}-1\right)_{m}\left(-2 a_{2}-1\right)_{m}}{\left(-3-2 \kappa-2 a_{2}-2 a_{3}\right)_{2 m+2} m !\left(2 a_{1}+1\right)_{m}} .
\end{aligned}
$$

Next calculate, recalling that $n=a_{1}+a_{2}+a_{3}+1$ and we obtain

$$
\begin{aligned}
& \frac{c\left(2 a_{1}+m+1,2 a_{3}+1-m, 2 a_{2}+1-m, m+1\right)}{c\left(2 a_{1}+m-1,2 a_{3}+1-m, 2 a_{2}+1-m, m+1\right)}=\frac{\left(\kappa+a_{1}+m\right)\left(2 a_{1}+2 m+1\right)}{(4 \kappa+2 n+1)(2 \kappa+n)}, \\
& \frac{c\left(2 a_{1}+m, 2 a_{3}+2-m, 2 a_{2}+2-m, m\right)}{c\left(2 a_{1}+m-1,2 a_{3}+1-m, 2 a_{2}+1-m, m+1\right)} \\
& =2 \frac{\left(\kappa+a_{2}+a_{3}+1-m\right)\left(\kappa+a_{2}+a_{3}+\frac{3}{2}-m\right)}{(4 \kappa+2 n+1)(2 \kappa+n)}, \\
& \frac{c\left(2 a_{1}+m+1,2 a_{3}+1-m, 2 a_{2}+1-m, m-1\right)}{c\left(2 a_{1}+m-1,2 a_{3}+1-m, 2 a_{2}+1-m, m+1\right)}=\frac{\left(2 \kappa+2 a_{2}+1\right)\left(2 \kappa+2 a_{3}+1\right)}{\left(2 a_{2}+1\right)\left(2 a_{3}+1\right)}, \\
& \frac{c\left(2 a_{1}+m, 2 a_{3}-m, 2 a_{2}+2-m, m\right)}{c\left(2 a_{1}+m-1,2 a_{3}+1-m, 2 a_{2}+1-m, m+1\right)}=\frac{2 \kappa+2 a_{3}+1}{2 a_{3}+1}, \\
& \frac{c\left(2 a_{1}+m, 2 a_{3}+2-m, 2 a_{2}-m, m\right)}{c\left(2 a_{1}+m-1,2 a_{3}+1-m, 2 a_{2}+1-m, m+1\right)}=\frac{2 \kappa+2 a_{2}+1}{2 a_{2}+1} .
\end{aligned}
$$

Write the right side of the summation formula in Theorem 6 in the form $d_{m}=A_{m} F\left(m ; \kappa+1, a_{1}, a_{2}, a_{3}\right)$. Then

$$
\begin{aligned}
\frac{A_{m}}{C_{m}} & =4 \kappa\left(\kappa+a_{1}+m\right)(4 \kappa+3 n+2) \frac{\left(2 a_{2}-m+1\right)\left(2 a_{3}-m+1\right)}{\left(2 a_{2}+1\right)\left(2 a_{3}+1\right)}, \\
\frac{A_{m-1}}{A_{m}} & =\frac{2 m\left(2 a_{1}+m\right)\left(\kappa+a_{2}+a_{3}+1-m\right)\left(\kappa+a_{2}+a_{3}+\frac{3}{2}-m\right)}{\left(\kappa+a_{1}+m\right)\left(2 a_{1}+2 m-1\right)\left(2 a_{2}-m+1\right)\left(2 a_{3}-m+1\right)} .
\end{aligned}
$$

The expression $\left(\frac{\left(-2 a_{2}-1\right)_{m}\left(-2 a_{3}-1\right)_{m}}{m !\left(2 a_{1}+1\right)_{m}} t_{m}+d_{m-1}-d_{m}\right) / C_{m}$ with $a_{i}$ replaced by $v_{i}$ (formal parameters instead of integers) for $1 \leq i \leq 3$ becomes the left side of the equation in the following result.

THEOREM 8. Suppose that $v_{1}, v_{2}, v_{3} \notin-\frac{1}{2}+\mathbb{N}_{0},\left(v_{0}=v_{1}+v_{2}+v_{3}+1\right)$ and $m \in$ $\mathbb{N}_{0}$. Then

$$
\begin{aligned}
& 2\left(2 v_{1}+1+2 m\right)\left(\kappa+v_{1}+m\right)\left(4 \kappa+v_{0}\right) F\left(m+1 ; \kappa, v_{1}, v_{2}+1, v_{3}+1\right) \\
& -4\left(4 \kappa+v_{0}\right)\left(\kappa+v_{2}+v_{3}+\frac{3}{2}-m\right)\left(\kappa+v_{2}+v_{3}+1-m\right) F\left(m ; \kappa, v_{1}, v_{2}+1, v_{3}+1\right) \\
& -\left(2 v_{1}+m\right)\left(4 \kappa+2 v_{0}+1\right)\left(2 \kappa+v_{0}\right) F\left(m+1 ; \kappa, v_{1}-1, v_{2}+1, v_{3}+1\right) \\
& \quad-\frac{\left(2 \kappa+2 v_{2}+1\right)\left(2 \kappa+2 v_{3}+1\right)}{\left(2 v_{2}+1\right)\left(2 v_{3}+1\right)} m\left(4 \kappa+2 v_{0}+1\right)\left(2 \kappa+v_{0}\right) F\left(m-1 ; \kappa, v_{1}+1, v_{2}, v_{3}\right)
\end{aligned}
$$




$$
\begin{aligned}
& +\frac{\left(2 \kappa+2 v_{3}+1\right)}{\left(2 v_{3}+1\right)}\left(2 v_{3}+1-m\right)\left(4 \kappa+2 v_{0}+1\right)\left(2 \kappa+v_{0}\right) F\left(m ; \kappa, v_{1}, v_{2}+1, v_{3}\right) \\
& +\frac{\left(2 \kappa+2 v_{2}+1\right)}{\left(2 v_{2}+1\right)}\left(2 v_{2}+1-m\right)\left(4 \kappa+2 v_{0}+1\right)\left(2 \kappa+v_{0}\right) F\left(m ; \kappa, v_{1}, v_{2}, v_{3}+1\right) \\
& +\frac{8 m \kappa\left(2 v_{1}+m\right)}{\left(2 v_{2}+1\right)\left(2 v_{3}+1\right)\left(2 v_{1}+2 m-1\right)}\left(\kappa+v_{2}+v_{3}+\frac{3}{2}-m\right)\left(\kappa+v_{2}+v_{3}+1-m\right) \\
& \times\left(4 \kappa+3 v_{0}+2\right) F\left(m-1 ; \kappa+1, v_{1}, v_{2}, v_{3}\right) \\
& -\frac{4 \kappa\left(2 v_{2}+1-m\right)\left(2 v_{3}+1-m\right)}{\left(2 v_{2}+1\right)\left(2 v_{3}+1\right)}\left(4 \kappa+3 v_{0}+2\right)\left(\kappa+v_{1}+m\right) F\left(m ; \kappa+1, v_{1}, v_{2}, v_{3}\right) \\
& =0 .
\end{aligned}
$$

Proof. The method is similar to the previous one; here we use $\gamma_{3}$ as the point of reference for the ratio calculations. Write the identity as $\sum_{n=1}^{8} \mu_{n} F\left(\gamma_{n}\right)=0$ and use the notation $t_{i}\left(\gamma_{n}\right)$ as before. As before $g_{i, k}(\kappa):=(\kappa+i)_{k}\left(-\kappa-v_{0}+i\right)_{k}$ (but here $v_{0}=\sum_{j=1}^{3} v_{j}+1$, different from the previous theorem). Set

$$
\begin{aligned}
& p_{i}(\kappa)=\sum_{n=1}^{2} \mu_{n} \frac{t_{i+1}\left(\gamma_{n}\right)}{t_{i}\left(\gamma_{3}\right)}+\mu_{3}+\sum_{n=4}^{8} \mu_{n} \frac{t_{i}\left(\gamma_{n}\right)}{t_{i}\left(\gamma_{3}\right)}, i \geq 1, \\
& p_{0}(\kappa)=\sum_{n=1}^{2} \mu_{n} t_{1}\left(\gamma_{n}\right)+\sum_{n=1}^{8} \mu_{n} .
\end{aligned}
$$

Then $p_{i}(\kappa)$ is a polynomial of degree 5 in $\kappa$. By computer algebra we find the coefficients $\left\{c_{i k}\right\}_{k=0}^{5}$, which are functions of $i, m, v_{1}, v_{2}, v_{3}$, so that

$$
p_{i}(\kappa)=c_{i, 0}+c_{i, 1} \kappa+\left(c_{i, 2}+c_{i, 3} \kappa\right) g_{i, 1}(\kappa)+\left(c_{i, 4}+c_{i, 5} \kappa\right) g_{i, 2}(\kappa) .
$$

Then the left side of the identity equals

$$
\begin{aligned}
& \sum_{i=0}^{\lfloor(m+1) / 2\rfloor} p_{i}(\kappa) g_{0, i}(\kappa) r_{i} \\
& =\sum_{i=0}^{\lfloor(m+1) / 2\rfloor} r_{i}\left(\left(c_{i, 0}+c_{i, 1} \kappa\right) g_{0, i}+\left(c_{i, 2}+c_{i, 3} \kappa\right) g_{0, i+1}(\kappa)+\left(c_{i, 4}+c_{i, 5} \kappa\right) g_{0, i+2}(\kappa)\right) \\
& =\sum_{i=0}^{\lfloor(m+1) / 2\rfloor+2} g_{0, i}\left(r_{i}\left(c_{i, 0}+c_{i, 1} \kappa\right)+r_{i-1}\left(c_{i-1,2}+c_{i-1,3} \kappa\right)+r_{i-2}\left(c_{i-2,4}+c_{i-2,5} \kappa\right)\right),
\end{aligned}
$$

where $r_{i}:=\frac{t_{i}\left(\gamma_{3}\right)}{g_{0, i}(\kappa)}$. The degree of $\kappa$ in $\mu_{1}$ and $\mu_{2}$ is 3 and thus $c_{0,4}+c_{0,5} \kappa$ agrees with the generic value of $c_{i, 4}+c_{i, 5} \kappa$ at $i=0$. In the range $2 \leq i \leq\left\lfloor\frac{m+1}{2}\right\rfloor$ the value of $\left(c_{i, 0}+c_{i, 1} \kappa\right)+\frac{r_{i-1}}{r_{i}}\left(c_{i-1,2}+c_{i-1,3} \kappa\right)+\frac{r_{i-2}}{r_{i}}\left(c_{i-2,4}+c_{i-2,5} \kappa\right)$ is found to be zero by symbolic computation. Also $c_{0,0}+c_{0,1} \kappa=0$ and $r_{1}\left(c_{1,0}+c_{1,1} \kappa\right)+r_{0}\left(c_{0,2}+c_{0,3} \kappa\right)=0$. The special cases at the top end of summation occur at $i=\frac{m}{2}$ or $i=\frac{m+1}{2}$ (for $m$ being even or odd, respectively) and in fact $c_{i, 4}+c_{i, 5} \kappa=0$ and

$$
\left(c_{i, 2}+c_{i, 3} \kappa\right)+\frac{r_{i-1}}{r_{i}}\left(c_{i-1,4}+c_{i-1,5} \kappa\right)=0
$$

for these values of $i$.

This completes the proof of Theorem 6 . 
4. Closing Comments. First we remark on the difficulty and complexity of the proof of the intertwining property. We speculate that one reason is that the measure on the linear transformations $\tau(q)$ is not uniquely defined. As was seen in the presentation it is only the integrals of $P_{a, b}^{c}(q)$ that matter. By Rösler's result [8] for each $x \in \mathbb{R}^{2}$ and $\kappa>0$ there is a positive Baire measure $\mu_{x}$ such that $V f(x)=\int f d \mu_{x}$ and the support of $\mu$ is contained in the closed convex hull of the $B_{2}$-orbit of $x$. The explicit measure in Theorem 4 requires $\kappa>\frac{3}{2}$ and is not positive. Make the substitutions $u=1-\varepsilon, \phi_{1}=$ $\phi_{2}=\frac{\pi}{2}, \psi_{1}=\pi-\varepsilon, \psi_{2}=\varepsilon$ (with $\left.0<\varepsilon<\frac{1}{4}\right)$; then $1+g_{0}(q)+g_{3}(q)=-1-\cos \theta+$ $2 \varepsilon+O\left(\varepsilon^{2}\right)$, and $\widetilde{g}_{0}=2 \cot ^{2} \varepsilon \cos \theta$, so that both terms are negative for small $\varepsilon$ and $\frac{\pi}{2}<\theta<\pi$. However the integral formula for the Bessel function $K^{0}(x, y)$ is positive and works for $\kappa>\frac{1}{2}$. It would be very interesting if one could develop explicit results for the Coxeter groups $A_{n}$ or $B_{n}$ for $n \geq 3$. The corresponding compact Lie groups $(U(n+1)$ and $S p(n)$, respectively) are fairly concrete but clearly we need more powerful techniques than we used here for $n=2$.

Secondly there is the two-parameter problem. The group $B_{2}$ allows two parameters in the associated differential-difference operators (see (1.1)). The approach to finding the measure $\mu$ by starting with the Lie group $S p(2)$ did not (as yet) help us in the twoparameter problem. This may be the hidden reason why the part of the intertwining formula associated with the representations of types 1 and 2 (realized on $x_{1} x_{2}$ and $x_{1}^{2}-x_{2}^{2}$ respectively) is so complicated (involving derivatives); we were somehow (heuristically) close to the kernels for $\kappa_{1}=\kappa+1, \kappa_{2}=\kappa$ and $\kappa_{1}=\kappa, \kappa_{2}=\kappa+1$.

Finally we comment on the role of computer algebra. We produced $K_{n}(x, y)$ for $n \leq 6$ by solving the equations $T_{i}^{x} K_{n}(x, y)=y_{i} K_{n-1}(x, y), i=1,2$. Then by use of the symmetrized kernels $\frac{1}{8} \sum_{w \in B_{2}} K_{n}(x w, y)$ we conjectured that the measure $\mu$ is the right one. Borrowing the polynomial $2\left(q_{1}+q_{4}\right)+q_{1} q_{4}-q_{2} q_{3}$ from the $S_{3}$ paper [4] quickly helped to expand the conjecture. The part of the intertwining operator dealing with types 1 and 2 (see Section 2.1) turned out to be puzzling. Eventually we had a formula for $V$ that worked for each $K_{n}(x, y)$ tried (up to $n=12$ ), but no proof. Then experimenting with $P_{a, b}^{c}(q)$ led to discovering the single-sum for $s(\alpha)$ (see Section 1.4). The proof itself depends on classical transformations of hypergeometric series. In turn the single sum expression (which had three free parameters) made feasible enough experiments to formulate the identity in Theorem 5. The term-by-term calculations which prove the contiguity relations in Section 3.3 would be very tedious without symbolic computation assistance, and much space would be needed to write down every intermediate step. However it is straightforward to verify these relations for any particular $m$ (magnitude depending on the size of the computer; $m=1 \ldots 6$ is not too big). There is some satisfaction in coaxing a symbolic computation system actually to prove a conjecture after it helped in the conjecture's formulation.

\section{REFERENCES}

1. W. Bailey, Generalized hypergeometric series (Cambridge University Press 1935).

2. C. Dunkl, Differential-difference operators associated to reflection groups, Trans. Amer. Math. Soc. 311 (1989), 167-183.

3. C. Dunk1, Operators commuting with Coxeter group actions on polynomials, in Invariant theory and tableaux (D. Stanton, ed.), (Springer-Verlag, 1990), 107-117.

4. C. Dunk1, Intertwining operators associated to the group $S_{3}$, Trans. Amer. Math. Soc. 347 (1995), 3347-3374. 
5. C. Dunkl, M. de Jeu, and E. Opdam, Singular polynomials for finite reflection groups, Trans. Amer. Math. Soc. 346 (1994), 237-256.

6. C. Dunkl and Y. Xu, Orthogonal polynomials of several variables, Encyclopaedia of Mathematics and its Applications 81 (Cambridge University Press, 2001).

7. S. Helgason, Groups and geometric analysis (Academic Press, New York, 1984). 463.

8. M. Rösler, Positivity of Dunkl's intertwining operator, Duke Math. J. 98 (1999), 445-

9. M. Rösler, Dunkl operators: theory and applications, Orthogonal polynomials and special functions (Leuven 2002), (E. Koelink and W. Van Assche, eds.), (Springer-Verlag, 2003), 93-135.

10. Y. Xu, A product formula for Jacobi polynomials, Special functions (Hong Kong 1999), (C. Dunkl, M. Ismail, R. Wong, eds.), (World Scientific, Singapore, 2000), 423-430. 